\title{
SUMO-specific protease 2 (SENP2) suppresses browning of white adipose tissue through $\mathrm{C} / \mathrm{EBP} \beta$ modulation
}

\author{
Ji Seon Lee ${ }^{1}$, Sehyun Chae ${ }^{2}$, Jinyan Nan ${ }^{1}$, Young Do Koo ${ }^{1}$, Seung-Ah Lee ${ }^{3}$, Young \\ Joo Park ${ }^{1,3}$, Daehee Hwang ${ }^{5}$, Weiping $\mathrm{Han}^{4}$, Young-Bum Kim ${ }^{3,6}$, Sung Soo Chung ${ }^{7 *}$, and \\ Kyong Soo Park ${ }^{1,3 *}$
}

${ }^{1}$ Department of Internal Medicine, Seoul National University College of Medicine, Seoul, Korea

${ }^{2}$ Korea Brain Bank, Korea Brain Research Institute, Daegu, Korea, Daegu, Korea

${ }^{3}$ Department of Molecular Medicine and Biopharmaceutical Sciences, Graduate

School of Convergence Science and Technology, College of Medicine, Seoul National University, Seoul, Korea

${ }^{4}$ Metabolism in Human Diseases Unit, Institute of Molecular and Cell Biology, Agency for Science, Technology and Research (A*STAR), Singapore, Singapore

${ }^{5}$ School of Biological Sciences, Seoul National University, Seoul, Korea

${ }^{6}$ Division of Endocrinology, Diabetes and Metabolism, Department of Medicine, Beth Israel

Deaconess Medical Center and Harvard Medical School, Boston, MA, USA

${ }^{7}$ Biomedical Research Institute, Seoul National University Hospital, Seoul, Korea

* Corresponding authors:

(Lead Contact) Kyong Soo Park, Department of Internal Medicine, Seoul National University College of Medicine, Seoul, Korea, 03080 Tel: 82-2-2072-2946, e-mail:kspark@ snu.ac.kr 
Sung Soo Chung, Biomedical Research Institute, Seoul National University Hospital, Seoul, Korea, 03082 Tel: 82-2-2072-1337, e-mail:suschung@snu.ac.kr

\section{Summary}

- Adipocyte-specific Senp2 knockout (Senp2-aKO) promotes WAT browning.

- Senp2-aKO mice are protected from high fat-induced obesity and insulin resistance.

- Reduction of Hoxc10 transcription is essential for SENP2-depletion-mediated beige adipogenesis in iWAT.

- Sumoylated C/EBP $\beta$ efficiently suppresses Hoxc10 transcription by recruiting the corepressor DAXX.

- $\mathrm{C} / \mathrm{EBP} \beta$ mediates thermogenic adipocyte differentiation by SENP2 depletion in both iWAT and eWAT. 


\section{Abstract}

SUMO-specific protease 2 (SENP2) is highly expressed in white adipose tissue (WAT) and plays an important role in the early stages of adipogenesis. To investigate the function of SENP2 in adipocytes, we generated adipocyte-specific Senp2 knock-out (Senp2-aKO) mice. Compared to wild-type mice, Senp2-aKO mice had reduced adipose tissue mass and smaller multi-locular adipocytes in inguinal WAT (iWAT). Body temperatures of Senp2-aKO mice were effectively regulated during cold exposure. Additionally, Senp2-aKO mice were resistant to high-fat-diet-induced obesity and insulin resistance and exhibited an increase in energy expenditure rates. Expression of thermogenic genes, including Ucpl, was significantly increased in iWAT (and less efficiently in epidydimal WAT [eWAT]) of Senp2aKO mice, suggesting that SENP2 depletion accelerates browning of WAT. Further, suppression of HOXC10 was essential for beige adipocyte formation in SENP2-deficient cells of iWAT, and Hoxc10 transcriptional suppression was mediated by C/EBP $\beta$, a direct target of SENP2. Sumoylated C/EBP $\beta$ efficiently inhibited Hoxc10 transcription through recruitment of the transcriptional co-repressor DAXX. Similarly, Senp2 knockdown using siRNAs during adipogenesis promoted thermogenic adipocyte differentiation of precursor cells in both iWAT and eWAT, and C/EBP $\beta$ was a common mediator. Together these results suggest that SENP2 plays critical role in white adipocyte differentiation by suppressing differentiation toward thermogenic adipocytes through modulation of $\mathrm{C} / \mathrm{EBP} \beta$ in both iWAT and eWAT. 


\section{Introduction}

Obesity is the primary contributing factor in several metabolic diseases and is caused by expansion of white adipose tissue (WAT) (1). White adipocytes consist of a single, large lipid droplet that stores energy, while brown adipocytes are responsible for adaptive thermogenesis $(2,3)$. Uncoupling protein 1 (UCP1), which is specifically expressed in brown adipocytes, catalyzes a proton leak across the mitochondrial membrane that results in heat generation (4). Recently, a third class of adipocytes, beige adipocytes, was identified as clusters of cells embedded in WAT. Beige adipocytes have multi-locular lipid droplets, similar to those of brown adipocytes, and contribute to thermogenesis through expression of UCP1 (5-7). Therefore, the induction of beige adipocytes is a promising therapeutic approach for obesity and diabetes.

Beige adipocyte formation in WAT is activated by cold temperature, $\beta$-adrenergic stimulation, chronic exercise, and various pharmacological treatments (8-13), and can also drive from mature white adipocytes (14). Conversely, beige adipocytes can switch to white adipocytes after an initial cold exposure ends but retain genetic memory and convert back to beige adipocytes upon a second cold stimulation (15). Another source of beige adipocytes is WAT progenitors. Several studies indicate that progenitor cells expressing specific markers, such as platelet-derived growth factor receptor beta, alpha smooth muscle actin and myosin heavy chain 11, generate beige adipocytes upon cold exposure (16-20). However, further studies are needed to understand the relative contributions of these cells to beige adipocyte formation.

Several transcription factors are involved in the adipose browning process $(21,22)$. First, peroxisome proliferator-activated receptor $\gamma(\operatorname{PPAR} \gamma)$ coactivator $1 \alpha(\mathrm{PGC} 1 \alpha)$ is induced by 
cold exposure and activates expression of thermogenic genes (23). Loss of PGC1 $\alpha$ in WAT reduces UCP1 expression and thermogenesis (24). PRDM16, a member of the PR/SET domain family, is highly expressed in brown adipose tissue (BAT), and controls brown fat development by binding and enhancing the transcriptional activities of CCAAT/enhancer binding protein $\beta(\mathrm{C} / \mathrm{EBP} \beta), \operatorname{PPAR} \gamma$ and $\mathrm{C} 2 \mathrm{H} 2$ zinc-finger protein $516(22,25)$. Subcutaneous WAT (scWAT) is very susceptible to browning, and PRDM16 is highly expressed in subcutaneous fat compared to visceral fat depots. Adipocyte-specific depletion of PRDM16 induces defects in scWAT thermogenesis and the conversion of subcutaneous to visceral fat (26). Further, overexpression of PRDM16 increases beige adipocytes in scWAT and induces protective effects against high-fat-diet (HFD)-induced obesity and insulin resistance (27).

Genes of the homeobox (Hox) family exhibit specific expression patterns in both WAT and BAT $(28,29)$. For example, Hoxc8, Hoxc9 and Hoxcl0 are highly expressed in WAT, while differentiation of human WAT progenitor cells into beige adipocytes is associated with downregulation of Hoxc8 (30). Expression of both Hoxc9 and Hoxcl0 is higher in subcutaneous than visceral adipose tissue and their expression degree is correlated with obesity (31). Furthermore, ectopic expression of Hoxc10 suppresses cold-induced browning in scWAT, suggesting that HOXC10 is a key negative regulator of browning (32).

In our previous studies, we showed that small ubiquitin-like modifier (SUMO)-specific protease 2 (SENP2) is required for adipogenesis and is involved in lipid metabolism in skeletal muscle. Specifically, knockdown of Senp2 expression in 3T3L1 preadipocytes inhibited adipogenesis through regulation of $\mathrm{C} / \mathrm{EBP} \beta$ stability during the early stages of adipogenesis (33). Senp2 expression was positively regulated by palmitate, and overexpression of Senp2 in skeletal muscle protected mice from HFD-induced obesity and 
insulin resistance by increasing fatty acid oxidation (FAO). SENP2 enhanced the transcription of genes encoding FAO-associated enzymes, including long-chain acyl-CoA synthetase 1 and carnitine-palmitoyl transferase $1 \mathrm{~b}$, through desumoylation-mediated activation of PPAR $\delta$ and PPAR $\gamma(34)$.

In the current study, we generated adipocyte-specific Senp2 knock out mice (Senp2-aKO) to thoroughly investigate the physiological roles of SENP2 in adipocytes. Interestingly, knockout of Senp2 using an adiponectin-Cre system enhanced browning of iWAT in the absence of cold exposure or treatment with a browning inducer. Additionally, Hoxc10 transcription was significantly reduced in the iWAT of Senp2-aKO mice, allowing the differentiation of white adipose progenitor cells toward thermogenic adipocytes. Furthermore, accumulation of sumoylated $\mathrm{C} / \mathrm{EBP} \beta$, a direct target of SENP2, efficiently suppressed Hoxc10 transcription. These results suggest that SENP2 negatively regulates browning and is important for maintaining white adipocyte identity during the differentiation of white adipose progenitor cells.

\section{Results}

\section{Generation of adipocyte-specific Senp2 knockout mice (Senp2-aKO)}

SENP2 expression was relatively high in white adipose tissues, inguinal WAT (iWAT) and epididymal WAT (eWAT), compared to BAT. Much lower levels of SENP2 were detected in muscle and liver, suggesting an important role of SENP2 in adipose tissues (Supplemental Fig. 1A). To investigate the function of SENP2 in adipocytes, we generated an adipocytespecific Senp2 knockout (Senp2-aKO) mouse model in which exon 3 of Senp2 was deleted by Cre recombinase driven by the adiponectin promoter (Fig. 1A). SENP2 protein and mRNA 
levels were dramatically reduced in iWAT, eWAT, and BAT in Senp2-aKO (aKO) mice compared to control mice (Fig. 1B and Supplemental Fig. 1B). When adipocytes were separated from the stromal vascular fractions (SVF) in iWAT, SENP2 protein levels were significantly reduced in adipocytes of Senp2-aKO mice compared with control mice, but were not changed in the SVF (Supplemental Fig. 1C). In addition, SENP2 expression in muscle or liver tissue was not changed in Senp2-aKO mice compared with control mice (Supplemental Fig. 1D).

\section{Adipocyte-specific depletion of SENP2 improved thermogenesis through accumulation of beige adipocytes in iWAT}

When mice were fed a normal chow diet, we observed no difference in body weights between control and Senp2-aKO mice (Fig. 1C). However, both iWAT and BAT mass significantly decreased in Senp2-aKO mice compared to control mice, whereas eWAT mass did not change (Fig. 1D). We observed no significant difference in glucose tolerance between control and Senp2-aKO mice, but Senp2-aKO mice had an improved insulin response (Fig. $1 \mathrm{E}$ and $1 \mathrm{~F})$. Histological analysis of adipose tissues from 12-week old mice showed that the average size of iWAT adipocytes from Senp2-aKO mice was smaller than that of control mice, whereas we found no change in eWAT adipocytes between the two groups (Fig. 1G and 1H). In addition, iWAT from Senp2-aKO mice had abundant multi-locular adipocytes and UCP1 expression (Fig. 1G and 1I-J). To compare adaptive thermogenesis, control and Senp2-aKO mice were exposed to cold conditions $\left(4^{\circ} \mathrm{C}\right)$. Body temperatures of both groups of mice dramatically decreased $30 \mathrm{~min}$ after cold exposure. However, unlike control mice, Senp2aKO mice gradually recovered their normal body temperature (Fig. 1K). Taken together, 
these results suggest that beige adipocytes accumulate in iWAT of Senp2-aKO mice and contribute to maintaining normal body temperature in a cold environment.

\section{Adipocyte-specific depletion of SENP2 protects mice from HFD-induced obesity and insulin resistance}

When mice were fed a HFD, body weight gain was attenuated in Senp2-aKO mice compared to control mice although there was no change in food intake by the mice (Fig. 2A and Supplemental Fig. 2A). The total body fat mass of the Senp2-aKO mice was significantly lower ( $40 \%$ reduction) than that of the control mice, but no change was observed in the lean mass or bone mineral density (Fig. 2B and Supplemental Fig. 2B). The average weight of each adipose tissue (eWAT, iWAT, and BAT) in Senp2-aKO mice was significantly lower than those in control mice, whereas we saw no change in the weight of other tissues, such as liver and muscle (Fig. 2C and Supplemental Fig. 2C).

To determine whether adipocyte-specific SENP2 depletion affected HFD-induced insulin resistance, glucose tolerance tests and insulin tolerance tests were performed after 12 or 13 weeks of HFD feeding. Senp2-aKO mice showed improved glucose tolerance, as well as decreased blood glucose levels in response to insulin (Fig. 2D and 2E). Serum insulin, triglyceride and free fatty acid levels were similar in control and Senp2-aKO mice (Supplemental Fig. 2D and 2E). In the Senp2-aKO mice, serum leptin and adiponectin levels were lower than those of control mice (Supplemental Fig. 2F), which was probably due to reduced fat mass. We also investigated morphological differences in adipose tissue between HFD-fed control and Senp2-aKO mice. Adipocyte size in WAT (eWAT and iWAT) was significantly reduced in Senp2-aKO mice (Fig. 2F and 2G). Further, we easily observed multi-locular droplets in iWAT of Senp2-aKO mice (Fig. 2F, right panel). In contrast, large 
lipid droplets were detected in BAT of Senp2-aKO (Fig. 2F).

Next, we measured energy expenditure rates to investigate the mechanism underlying the effects of Senp2 knockout and found that $\mathrm{O}_{2}$ consumption and heat production rates increased in Senp2-aKO mice during both light and dark cycles (Fig. 2H-2K). These results suggest that adipocyte-specific SENP2 depletion elevates energy expenditure, which results in protection of mice from HFD-induced obesity and insulin resistance.

\section{SENP2 depletion promotes expression of thermogenic genes in iWAT and eWAT}

The iWAT gene expression profiles of HFD-fed control and Senp2-aKO mice were analyzed using micro-arrays and quantitative PCR (qPCR) analysis. Genes involved in negative regulation of lipid storage, heat generation and brown fat differentiation were upregulated in iWAT of Senp2-aKO mice, whereas genes related to steroid or lipid biosynthesis were down-regulated in Senp2-aKO iWAT (Fig. 3A). While the mRNA levels of genes abundantly expressed in white adipocytes were reduced or unchanged, expression of brownadipocyte-specific genes, such as Ucp1, Cidea, Elovl3, and Dio2, was significantly elevated in iWAT of Senp2-aKO mice (Fig. 3B and 3C). The difference in expression of BAT-specific genes between HFD-fed control and Senp2-aKO mice was greater than the difference seen in mice fed a normal chow diet (Fig. 3C and 3D; 30-fold vs. 4-fold for Ucp1). The mRNA levels of mitochondrial oxidative-phosphorylation-related genes, such as Cox7al and Cox8b, were also higher in iWAT of Senp2-aKO mice (Fig. 3C and 3D). These results indicate the efficient accumulation of beige adipocytes in iWAT by SENP2 deficiency.

We compared the expression levels of several transcription factors related to adipogenesis and browning and saw no significant change of those tested, including Prdm16, an important 
regulator that induces browning (Fig. 3C and 3D, right panel and Supplemental Fig. 3A). However, expression of Hoxc10, a known negative regulator of browning, was significantly reduced in iWAT of HFD-fed Senp2-aKO mice (Fig. 3C) which was consistently observed under normal chow-diet feeding (Fig. 3D), suggesting the possibility that reduced expression of Hoxc10 accelerates browning of iWAT in Senp2-aKO mice.

We also compared gene expression patterns of eWAT in control and Senp2-aKO mice. Expression of Ucp1, Elovl3 and Dio2 in eWAT of Senp2-aKO mice was higher than that of control mice fed either a normal chow diet or HFD (9-15 fold for Ucp1, 2-5 fold for Elovl3 and Dio2) (Supplemental Fig. 3B and 3C). Unlike iWAT, Hoxc10 mRNA levels in eWAT were very low (Supplemental Fig. 3D) and were not affected by Senp2 knockout (Supplemental Fig. 3B and 3C). Although beige-like adipocyte depots were not observed in eWAT based on the H\&E staining results (Fig. 2F), the increased thermogenic gene expression seen in eWAT may partially contribute to alleviating HFD-induced obesity and insulin resistance in Senp2-aKO mice.

\section{SENP2 depletion facilitates differentiation of white adipose progenitor cells into thermogenic adipocytes}

We found that Senp2 knockout results in thermogenic gene expression in white adipocytes, therefore we next accessed to figure out the mechanism to induce thermogenic adipocytes. The SVF was isolated from iWAT of control and Senp2-aKO mice, and then differentiation into adipocytes was induced (Fig. 4A). After differentiation, lipid droplets were stained with Oil-Red O (Fig. 4B). Similar to the gene expression patterns in iWAT, expression of brownadipocyte-specific genes was higher (5-6 fold for Ucp1 and Cidea; 2-3 fold for Elovl3, Dio2 and $P g c l \alpha$ ), while Hoxc10 expression was lower in SVF-derived adipocytes from Senp2- 
aKO mice (Fig. 4C). To confirm the thermogenic response of the SENP2-deficient adipocytes, cells were treated with the $\beta 3$-adrenergic receptor agonist CL316243. Ucpl and Pgcl $\alpha$ mRNA levels were rapidly increased upon CL316243 treatment in SENP2-deficient adipocytes but not in control adipocytes (Fig. 4D). Increased UCP1 expression was confirmed by western blot analysis (Fig. 4E). We also compared gene expression patterns at several time points during differentiation of the progenitor cells into adipocytes. In the cells isolated from Senp2-aKO mice, Senp2 mRNA levels were decreased at day 4, likely responding to increased adiponectin. Hoxc10 mRNA levels also decreased, whereas Ucpl mRNA levels gradually increased in SENP2-deficeint cells (Fig. 4F). Similar to Ucp1, expression of Cidea, Elovl3 and Pgcl $\alpha$ also gradually increased in SENP2-deficient cells during adipogenesis (Supplemental Fig. 4). These results suggest that SENP2 depletion during adipogenesis of white adipose progenitor cells changes the direction of differentiation toward beige or beige-like adipocytes.

\section{Suppression of Hoxc10 is necessary for Senp2 knockout-induced beige adipogenesis in iWAT}

HOXC10 is a known negative regulator of browning (32), and Hoxc10 expression was suppressed in iWAT of Senp2-aKO mice and their adipocytes (Fig. 3C-D and 4C). Therefore, we tested whether Hoxc10 overexpression inhibited Senp2-knockout-induced beige adipocyte differentiation. Five days after the induction of adipocyte differentiation in iWAT SVF cells of control or Senp2-aKO mice, cells were infected with adeno-associated virus expressing Hoxc10 (AAV-Hoxc10) (Fig. 5A). We confirmed Hoxc10 overexpression at both the mRNA and protein levels (Fig. 5B and Supplemental Fig. 5A). UCP1 induction in SENP2-deficient adipocytes was completely inhibited by overexpression of HOXC10 (Fig. 
5B). In addition to Ucpl, expression of other brown-adipocyte-specific genes, including Cidea, Elovl3, Dio2, and Pgcla, were not induced in SENP2-deficient adipocytes after Hoxc10 overexpression (Fig. 5C), indicating that suppression of Hoxc10 expression is necessary for beige adipocyte differentiation in iWAT of Senp2-aKO mice.

Next, we knocked down expression of the transcription factors PPAR $\gamma, \mathrm{C} / \mathrm{EBP} \beta, \mathrm{PGC} 1 \alpha$, and PRDM16 using specific siRNAs at day 5 of differentiation to investigate their involvement in SENP2-depletion-induced beige adipocyte differentiation (Fig. 5D and Supplemental Fig. 5B-5C). Although Ppar $\gamma$ knockdown affected expression of some brown adipocyte-specific genes such as Ucp1, Cidea and Pgcla (Fig. 5E-5F and Supplemental Fig. 5C), clearer changes were observed with $C / e b p \beta$ knockdown, which completely abrogated increased mRNA levels of Ucpl, Cidea, Elovl3, Pgcla, and Prdm16 and reduced Hoxc10 mRNA induced by SENP2 depletion. In contrast, the effects of Pgcl $\alpha$ or Prdm16 knockdown were not significant (Fig. 5E-5H and Supplemental Fig. 5C). The impact of C/ebp $\beta$ knockdown on UCP1 and HOXC10 was confirmed by western blot analysis (Fig. 5I), further highlighting $\mathrm{C} / \mathrm{EBP} \beta$ as a key factor involved in beige adipocyte differentiation induced by SENP2 deficiency in iWAT.

\section{Sumoylated C/EBPß negatively regulates Hoxc10 transcription}

Our results showed that suppression of Hoxc10 was necessary for beige cell differentiation induced by SENP2 deficiency in iWAT and that C/EBP $\beta$ mediated Hoxc10 suppression. Therefore, we next investigated how Hoxc10 transcription is regulated by $\mathrm{C} / \mathrm{EBP} \beta$. Several studies have reported that $\mathrm{C} / \mathrm{EBP} \beta$ can be sumoylated (35), which decreases its activity (36). C/EBP $\beta$ enhances promoter activities of various target genes; however, a few reports indicate that $\mathrm{C} / \mathrm{EBP} \beta$ also represses transcription of some target genes $(37,38)$. To 
test whether C/EBP $\beta$ directly suppresses Hoxc10 transcription, we used transient transfection and a reporter assay system. Briefly, Cos7 cells were transfected with Hoxc10 (-1567)-Luc and expression vectors for wild-type C/EBP $\beta$ (C/EBP $\beta$ WT) or C/EBP $\beta$ K133R, the latter of which contains a lysine-to-arginine mutation at the SUMO conjugation site, K133. Similar levels of $\mathrm{C} / \mathrm{EBP} \beta \mathrm{WT}$ and $\mathrm{C} / \mathrm{EBP} \beta \mathrm{K} 133 \mathrm{R}$ were expressed in a dose-dependent manner (Supplemental Fig. 6). C/EBP $\beta$ WT, but not K133R, reduced the luciferase activity of Hoxc10 (-1567)-Luc, and co-expression of SUMO and UBC9, a SUMO-conjugation enzyme, amplified the inhibitory activity of C/EBP $\beta$ WT (Fig. 6A). When SENP2 expression vectors were co-transfected, the C/EBP $\beta$-mediated suppression of luciferase activity was diminished in a dose-dependent manner, whereas we observed no effect in C/EBP $\beta$ K133R (Fig. 6B). Sumoylated C/EBP $\beta$ was detected in cells transfected with C/EBP $\beta$ WT, but not K133R, and SUMO-conjugated C/EBP $\beta$ gradually decreased as SENP2 increased (Fig. 6C).

To generate constitutive sumoylated $\mathrm{C} / \mathrm{EBP} \beta$, we constructed expression vectors encoding SUMO fused to the N-terminus of C/EBP $\beta$ WT or K133R and confirmed expression of the SUMO-fused proteins by western blot analysis (Fig. 6D). SUMO-fused C/EBP $\beta$ K133R, as well as SUMO-fused C/EBP $\beta$ WT, effectively suppressed the Hoxc10 promoter activity in the absence of UBC9 or SUMO overexpression (Fig. 6E). These results suggest that sumoylated C/EBP $\beta$ suppresses transcription of Hoxcl0.

\section{Sumoylated C/EBPß inhibits Hoxc10 transcription by recruiting the co-repressor death domain-associated protein (DAXX)}

To identify a cis-acting element for $\mathrm{C} / \mathrm{EBP} \beta$ binding in the Hoxcl0 promoter, we generated a deletion construct of the Hoxc10 promoter [Hoxc10 (-908)-Luc]. C/EBP $\beta$ WT did not suppress the luciferase activity of Hoxc10 (-908)-Luc (Fig. 6F, upper panel). We induced mutations at a putative C/EBP $\beta$ binding site at -1030 [Hoxc10 (-1567mt)-Luc]. C/EBP $\beta$ WT 
did not inhibit the promoter activity of Hoxc10 (-1567mt)-Luc (Fig. 6F, lower panel), demonstrating that $\mathrm{C} / \mathrm{EBP} \beta$ suppresses Hoxc10 transcription through the $\mathrm{C} / \mathrm{EBP} \beta$ binding site.

$\mathrm{C} / \mathrm{EBP} \beta$ represses promoter activity of target genes through interaction with corepressors, such as silencing mediator of retinoid and thyroid hormone receptors (SMRT) $(38,39)$. Therefore, we hypothesized that sumoylated C/EBP $\beta$ recruits a corepressor to the transcriptional complex to inhibit transcription of Hoxc10. To test this hypothesis, we performed chromatin immunoprecipitation (ChIP) using adipocytes derived from iWAT SVF of control or Senp2-aKO mice with an antibody against SMRT. We also used an anti-DAXX antibody for ChIP, as DAXX has a SUMO-interacting motif and mediates SUMO-dependent transcriptional repression of glucocorticoid receptor and cAMP response element binding protein (40-42). Binding of Daxx, but not SMRT, to the C/EBP $\beta$ binding site of the Hoxc10 promoter was stronger in SENP2-deficient adipocytes than in control adipocytes, without impacting $\mathrm{C} / \mathrm{EBP} \beta$ binding to the promoter (Fig. 6G). These results suggest that sumoylated $\mathrm{C} / \mathrm{EBP} \beta$, which is increased by SENP2 depletion, recruits DAXX to suppress Hoxc10 transcription. To confirm that sumoylated C/EBP $\beta$ efficiently interacts with DAXX, we performed co-immunoprecipitation and found that C/EBP $\beta$ WT interacted with DAXX to a greater extent than $\mathrm{C} / \mathrm{EBP} \beta \mathrm{K} 133 \mathrm{R}$ (Fig. $6 \mathrm{H}$ ), suggesting that sumoylation of $\mathrm{C} / \mathrm{EBP} \beta$ promotes interaction between $\mathrm{C} / \mathrm{EBP} \beta$ and $\mathrm{DAXX}$. In summary, SENP2 facilitates desumoylation of C/EBP $\beta$, which results in high levels of Hoxc10 transcription in control white adipocytes. However, when SENP2 is depleted, the sumoylated form of C/EBP $\beta$ increases and recruits DAXX, resulting in repression of Hoxc10 transcription in iWAT adipocytes (Fig. 6I).

SENP2 plays an important role in maintaining white adipocyte identity during 


\section{adipogenesis through modulation of C/EBPß in both iWAT and eWAT}

We next confirmed the effect of SENP2 depletion on the differentiation of adipose progenitor cells using Senp2 siRNAs (Fig. 7A). When SVF from iWAT of control mice was treated with siRNAs targeting Senp2 at day 3 of differentiation, the mRNA levels of brown adipocyte-specific genes such as Ucpl, Cidea, and Elovl3, were significantly increased (Fig. 7B). We also detected a reduction of Hoxc10 mRNAs. In addition, Hoxc10 overexpression by infection of AAV-Hoxc10 ameliorated the effects of Senp2 knockdown (Fig. 7C and Supplemental Fig. 7A). We next tested whether SENP2 depletion also stimulates mature white adipocytes to acquire the features of beige adipocytes. When fully differentiated adipocytes (day 7 of differentiation) were transfected with siRNA targeting Senp2, we observed little or no change in the expression of brown adipocyte markers (Fig. 7B). In addition to iWAT browning, thermogenesis-related gene expression was elevated in eWATs of Senp2-aKO mice (Supplemental Fig. 3B and 3C), although the expression levels were lower than those in iWATs. We therefore tested whether Senp2 knockdown induces expression of thermogenesis-related genes during differentiation of eWAT SVF (Fig. 7D). Similar to iWAT, Senp2 knockdown by transfection with siRNA at day 3, but not at day 7, slightly increased Ucpl and Cidea expression (Fig. 7E). These results suggest that SENP2-depletion-induced beige or thermogenic adipocyte formation mainly occurs during differentiation of adipose progenitor cells rather than in mature white adipocytes.

We also tested whether C/EBP $\beta$ mediates thermogenic gene induction by Senp2 knockout in eWAT (Fig. 7F and Supplemental Fig. 7B). Ucpl and Cidea transcripts were increased in the adipocytes derived from eWAT SVF of Senp2-aKO mice, and knockdown of C/EBP $\beta$ suppressed those increases (Fig. 7G). These results demonstrate that C/EBP $\beta$ is also involved in thermogenic gene induction in eWAT of Senp2-aKO mice. Unlike iWAT, Hoxc10 mRNA 
levels were very low in eWAT (Supplemental Fig. 3D), and Hoxc10 transcription was not affected by Senp2 knockout in eWAT (Supplemental Fig. 7B). Therefore, another factor that highly expressed in eWAT may act as a functional substitute for HOXC10.

To profile changes in the expression of various gene induced by SENP2 depletion in relation to C/EBP $\beta$, we performed RNA sequencing after adipocyte differentiation of SVF from eWAT and iWAT of control or Senp2-aKO mice in the presence or absence of C/EBP $\beta$ knockdown (Supplemental Fig. 8A). We focused on expression patterns of various transcription factors. Several transcription factors whose expression was increased in both Senp2-aKO eWAT and iWAT in a C/EBP $\beta$-dependent manner, such as PGC1 $\alpha$, STAT5, ZBTB16, and NOTCH1 (Fig. 7H, group A), have been reported to positively regulate beige adipocyte formation and thermogenesis (43-45). In addition to Pgcl $\alpha$, transcription of Stat5 and Zbtb16 was also suppressed by Hoxc10 overexpression in iWAT adipocytes (Supplemental Fig. 8B), which supports the C/EBP $\beta$-HOXC10-transcription factor axis for mediating browning in iWAT. In contrast, expression of some transcription factors, such as SMAD3, NOTCH 4, and SKIL, was decreased in eWAT and iWAT of Senp2-aKO but was recovered by knockdown of $\mathrm{C} / \mathrm{EBP} \beta$ (Fig. 7H, group $\mathrm{B}$ ). Interestingly, these transcription factors have been reported to promote white adipocyte differentiation and inhibit thermogenesis $(46,47)$. Therefore, we suggest that $\mathrm{C} / \mathrm{EBP} \beta$ is a master regulator of the expression of many transcription factors involved in adipocyte differentiation and browning in both iWAT and eWAT and that the direction of differentiation between white and beige or beige-like adipocytes can be determined by modulating SUMO modification of C/EBP $\beta$. In other words, SENP2 plays a role in maintaining white adipocyte identity during differentiation of white adipose progenitor cells by modulation of C/EBP (Fig. 7I).

\section{Discussion}


We have previously shown that SENP2 plays an important role in the early stages of adipocyte differentiation in vitro. To identify other possible functions of SENP2 in adipocytes, we generated adipocyte-specific Senp2 knockout mice using an adiponectin-Cre system in the current study. Here, we demonstrated that SENP2 depletion significantly reduced fat accumulation and facilitated formation of beige adipocytes in WAT. In addition, SENP2 depletion during adipogenesis caused white adipose progenitor cells to differentiate toward beige adipocytes. Therefore, SENP2 has dual functions in white adipocytes: regulating initial adipogenesis and maintaining white adipocyte identity throughout differentiation. Interestingly, we identified C/EBP $\beta$ as a direct target of SENP2 that modulates both of these functions. SENP2 stabilized C/EBP $\beta$ through desumoylation at the early stages of adipogenesis (33) and abolished sumoylated C/EBP $\beta$-mediated suppression of browning inhibitors, such as Hoxc10, which maintained white adipocyte identity during differentiation.

Traditionally, C/EBP $\beta$ induces transcription of various target genes, although some reports have described $\mathrm{C} / \mathrm{EBP} \beta$-mediated transcriptional repression. Several co-repressors, including SMRT, SWItch/Sucrose Non-Fementable, and CA150, interact with C/EBP $\beta$ and repress target gene expression $(38,39,48)$. In this study, we demonstrated that a co-repressor, DAXX, interacted with $\mathrm{C} / \mathrm{EBP} \beta$ and repressed Hoxc10 transcription. DAXX has a known SUMO-interacting motif (42), and we observed that the DAXX binding of C/EBP $\beta$ was increased by $\mathrm{C} / \mathrm{EBP} \beta$ sumoylation. Therefore, we suggest that sumoylated $\mathrm{C} / \mathrm{EBP} \beta$ effectively recruits DAXX, resulting in suppression of Hoxc10 transcription (Figure 6I). Further experiments will be required to determine whether the C/EBP $\beta$-DAXX complex represses transcription of other genes whose expression was downregulated in white adipocytes of Senp2-aKO mice.

In addition to Hoxc10, expression of several other transcription factors was modulated in WAT by Senp2 knockout. Although our data demonstrated that Hoxc10 suppression was 
important for browning in iWAT of Senp2-aKO mice, other transcription factors may also contribute to browning. As such, the transcription of several Hox genes, including Hoxc6, Hoxc8, and Hoxb5, was also downregulated in iWAT of Senp2-aKO mice (Supplemental Fig. 3A). Notably, these genes are highly expressed in white adipocytes, and that HOXC8 is known to inhibit brown adipocyte differentiation (49). Therefore, several HOX factors may work together to regulate expression of genes that determine brown or white adipocyte differentiation. Although the mechanism by which HOXC10 inhibits beige adipocyte differentiation in WAT has not been studied in detail, our RNA-seq analysis (Fig. 7H and Supplemental Fig. 8B) showed that HOXC10 inhibits expression of several transcription factors involved in beige adipogenesis, such as Pgcl $\alpha$ and Stat5a, suggesting that HOXC10 suppresses expression of upstream transcription factors, rather than directly regulating expression of thermogenic genes, such as Ucpl. However, further promoter analyses are required to determine how $\mathrm{HOXC10}$ regulates expression of these transcription factors. Unlike iWAT, Hoxc10 expression is very low in eWAT; therefore, a different C/EBP $\beta$ downstream factor(s) may suppress thermogenic activation in eWAT.

Our results suggest that the direction of adipocyte differentiation can be controlled through post-translational modification of $\mathrm{C} / \mathrm{EBP} \beta$ in iWAT and eWAT even though iWAT and eWAT adipocytes originate from different cell lineages. WAT consists of adipocytes derived from several types of precursor cells, while beige adipocyte differentiation is induced in specific lineages $(20,50,51)$. Therefore, determining which cell lineage(s) is induced to differentiate into beige adipocytes as a result of Senp2 knockout is of great interest. C/EBP $\beta$ is known to be involved in both white and brown adipocyte differentiation (22) and is thought to regulate the expression of $U c p l$ and other thermogenic genes by directly binding to their promoters (52). Therefore, these varied and sometimes contradictory roles of C/EBP $\beta$ may be accomplished by precise modulation of $\mathrm{C} / \mathrm{EBP} \beta$ through post-translational modification such 
as sumoylation.

One study demonstrated that SENP2 deficiency reduced adipose lipid storage and increased fat accumulation in other tissues, such as the liver and muscle tissues, which resulted in insulin resistance (53). With the exception of decreased fat mass, however, those results were different from our data due to deficiency of browning effects. In that study, Senp2-aKO mice were generated by deleting exons 13 and 14 from Senp2, which encode the C-terminus of SENP2. In our construct, however, exon 3 was deleted, and we confirmed that no truncated form of SENP2 was produced in adipose tissue of Senp2-aKO mice (Supplemental Fig. 1C). Therefore, the contradictory results of the two studies could be a consequence of different knockout strategies.

BAT mass was dramatically reduced in Senp2-aKO mice fed either a normal chow diet or HFD. We observed enlarged lipid droplets in BAT of HFD-fed Senp2-aKO mice, suggesting that the function of brown fat in Senp2-aKO mice was impaired. A recent report demonstrated that SENP2 is important for brown adipose development using Senp2-BAT knockout mice generated with myogenic factor 5-Cre (54). Therefore, SENP2 depletion might directly affect brown adipocyte differentiation and function, as Senp2 transcription was downregulated in BAT of Senp2-aKO mice. However, BAT reduction in Senp2-aKO mice may also be a secondary effect of beige adipocyte accumulation in WAT. Further study using brown adipocyte-specific Senp2 knockout mice generated using Ucpl-Cre will clarify the function of SENP2 in mature brown adipocytes.

To summarize, SENP2, highly expressed in white adipocytes, inhibits browning of WAT by modulation of $\mathrm{C} / \mathrm{EBP} \beta$. Inducing browning of WAT by inactivation of SENP2 or downregulation of Senp2 expression can be a therapeutic approach to ameliorate obesity and metabolic syndromes including diabetes. 


\section{Materials and Methods}

\section{Generation of adipocyte-specific Senp2 knockout mice}

Senp $2^{\text {flox/+ }}$ mice were generated by the inGenious Targeting Laboratory (Stony Brook, NY, USA) as described previously (55). Senp2 adipocyte specific-knockout (Senp2-aKO) mice were generated by a serial mating Senp $2^{\text {floxflox }}$ with adiponectin-Cre transgenic mice (Jackson lab).

\section{Mice and Metabolic analysis}

All aspects of animal care and experiments were conducted in accordance with the Guide for the Care and Use of Laboratory Animals of the National Institutes of Health, and approved by the Institutional Animal Care and Use Committee of Seoul National University Bundang Hospital, Korea (Permit Number: BA1403-149/013-01). All animals were housed at $22-24^{\circ} \mathrm{C}$, with a 12:12-h light-dark cycle and ad libitum access to standard pelleted chow or high-fat diet, (HFD; 58 kcal\% fat w/sucrose, D12331; Research Diets, New Brunswick, NJ, USA) and water. Eight week-old mice were fed a HFD for 12 weeks. Body weight was measured weekly, and body composition was measured by dual-energy X-ray absorptiometry (DXA) and LUNAR Prodigy scanner with software version 8.10 (GE Healthcare, PA, USA). Mice were maintained at room temperature or $4^{\circ} \mathrm{C}$ when indicated, and body temperatures were measured rectally using a digital thermometer. Metabolic cage experiments were conducted using a Comprehensive Lab Animal Monitoring System (CLAMS; Columbus Instruments, Columbus, OH, USA). Food and water intake were recorded weekly during HFD feeding. 
For the glucose tolerance test (GTT), glucose (1 $\mathrm{g} / \mathrm{kg}$ body weight) was treated by intraperitoneal (i.p.) injection after $16 \mathrm{~h}$ starvation. For the insulin tolerance test (ITT), mice fasted for $6 \mathrm{~h}$ were injected with human insulin (1 U/kg body weight). Serum glucose levels were determined using an OneTouch Ultra glucometer (LifeScan, Milpitas, CA, USA). Serum insulin (ALPCO, NA, USA), leptin, (Millipore, Billerica, MA, USA) and adiponectin (Millipore) were measured by enzyme-linked immunosorbent assays. Triacylglycerol (Cayman Chemical, Ann Arbor, MI, USA) and free fatty acid (Wako chemicals, Richmond, VA, USA) levels were determined using colorimetric assays.

\section{Histological analysis}

Tissues were fixed in $4 \%$ formaldehyde, embedded in paraffin and sectioned. Sections were subjected to hematoxylin and eosin (H\&E) staining. Immunohistochemistry was performed with antibodies against UCP1 (Abcam, Cambridge, UK). Lipid droplet diameters were measured using Leica software. 100-150 adipocytes per mouse were measured.

\section{Microarray experiments}

The mRNA profiles of iWATs from control and Senp2-aKO mice were analyzed using the Agilent mouse 8x60k chip, which includes 62,976 probes corresponding to 21,895 annotated genes (Agilent, Santa Clara, CA, USA). Gene expression profiles were generated from four replicates in each condition. Total RNA was extracted, and the integrity of the total RNA was analyzed using an Agilent 2100 Bioanalyzer. RNA integrity numbers for all samples were larger than 7.3. The in vitro transcription was then performed to generate cRNA, which was hybridized onto each array, according to the manufacturer's instructions. The array was 
scanned using a SureScan Microarray Scanner (Agilent).

\section{Identification of differentially expressed genes (DEGs)}

The intensities of the probes from the arrays were converted to $\log _{2}$-intensities and then normalized using the quantile normalization method (56). To identify DEGs, an integrative statistical hypothesis tests was performed as previously described (57). Briefly, for each gene, a T-statistic value was calculated using Student's t-test and also a $\log _{2}$-fold change in the comparison of Senp2-aKO versus control was calculated. We then estimated empirical distributions of T-statistic values and $\log _{2}$-fold changes for the null hypothesis (i.e. the genes are not differentially expressed) by performing all possible random permutations of the eight samples. Using the estimated empirical distributions, we computed adjusted p-values for the two tests for each gene and then combined these p-values with Stouffer's method (58). Finally, DEGs was identified as the genes with combined p-values $\leq 0.01$ and absolute $\log _{2}$ fold changes $\geq 0.58$ (1.5-fold change).

\section{Functional enrichment analysis}

Functional enrichment analysis was performed using DAVID software to identify gene ontology biological processes (GOBPs) enriched by the DEGs (59). The GOBPs enriched by the up- and down-regulated genes were identified as the ones with the enrichment P-value < 0.05 calculated by DAVID software.

mRNA sequencing and data analysis 
Total RNA was obtained from WATs (eWAT and iWAT) of control or Senp2-aKO mice in the absence or presence of $C / e b p \beta$ knockdown. The integrity of the total RNA was analyzed using an Agilent 2100 Bioanalyzer. RNA integrity numbers for all samples were larger than 8.9. Poly(A) mRNA isolation from total RNA and subsequent fragmentation were performed using the NEBNext Ultra II Directional RNA-seq Kit, according to the manufacturer's instructions (New England Biolabs, Ipswich, MA, USA). The adaptor ligated libraries were sequenced using an Illumina HiSeq $\mathrm{X}$ Ten (Ebiogen, Korea). mRNA sequencing was performed for three independent replicates under each condition. From the resulting read sequences for each sample, adapter sequences (TruSeq universal and indexed adapters) were removed using the cutadapt software (version 2.7). The remaining reads were then aligned to the Mus musculus reference genome (GRCm38) using TopHat2 software (version 2.1.1) with default parameters (60).After the alignment, we counted the numbers of reads mapped to the gene features (GTF file of GRCm38.91) using HTSeq (61).Read counts for the samples in each condition were then normalized using the trimmed mean of M-values (TMM) normalization method in the edgeR package (62).

\section{Identification of the differential expression patterns by non-negative matrix factorization (NMF) clustering}

To effectively identify the major clusters of genes showing altered expression patterns in multiple comparisons, we performed NMF clustering as previously described (63). For NMF analysis, we calculated $\log _{2}$-fold changes of each gene in the following comparisons using the normalized read counts in eWAT or iWAT samples: siClebp $\beta$ cont/siNS_cont, siNS_Senp2aKO/siNS_cont, and siClebp $\beta \_S e n p 2-a K O / s i N S \_c o n t$. These $\log _{2}$-fold changes were combined into a fold change matrix. Using the fold change matrix, we then performed NMF 
clustering and selected the genes defining the individual clusters with the following parameters as previously described (63): the number of clusters $(k)=50$ and p-value cutoff $=$ 0.01 for the gene selection.

\section{Real time qPCR}

Total RNAs were isolated using TRIzol (Invitrogen, MA, USA) according to the manufacturer's instruction. Real-time qPCR was performed (in duplicates) using the SYBRmaster mix (Takara, Otsu, Shiga, Japan) and the ABI 7500 Real-time PCR system (Applied Biosystem, CA, USA). Each cycle threshold (Ct) value was subtracted from $\mathrm{Ct}$ value of GAPDH or $\beta$-actin $(\triangle \mathrm{Ct})$, and then subtracted from the value of each control set $(\triangle \triangle \mathrm{Ct})$.

Relative mRNA levels were expressed as $2^{-\Delta \Delta \mathrm{Ct}}$. Primer sequences are listed in Supplemental Table 1.

\section{Isolation of SVF and in vitro differentiation}

Stromal vascular fraction (SVF) cells were isolated from the iWAT of 8 to12 week-old mice. Tissues were minced in digestion buffer (100 mmol/l HEPES pH7.4, $120 \mathrm{mmol} / \mathrm{l} \mathrm{NaCl}, 50$ $\mathrm{mmol} / \mathrm{l} \mathrm{KCl}, 5 \mathrm{mmol} / \mathrm{l}$ glucose, $1 \mathrm{mmol} / \mathrm{CaCl}_{2}, 1.5 \% \mathrm{BSA}$-fatty acid free, and $1 \mathrm{mg} / \mathrm{ml}$ collagenase II), and incubated at $37^{\circ} \mathrm{C}$ with shaking for $1 \mathrm{~h}$. Large particles were removed using a $100 \mu \mathrm{m}$ cell strainer, and $20 \mathrm{ml}$ Hank's buffered salt solution (HBSS, Gibco Life Technolosies, Carlsbad, CA, USA) supplemented with $10 \%$ fetal bovine serum (FBS, Gibco Life Technolosies) and $10 \mathrm{mM}$ HEPES was added to filtrates. The filtrates were centrifuged at 2,500 $\mathrm{rpm}$ for $10 \mathrm{~min}$ to remove floating adipocytes. The pelleted SVF cells were 
suspended in Dulbecco's modified Eagle's medium (DMEM, Gibco Life Technolosies) supplemented with $10 \%$ FBS, and seeded in 12-well plates. Adipocyte differentiation of confluent SVF cells was induced by treatment with DMI $(1 \mu \mathrm{mol} / 1$ dexamethasone, 0.5 $\mathrm{mmol} / \mathrm{l}$ isobutylmethyxanthine and $0.1 \mathrm{mg} / \mathrm{ml}$ insulin) in DMEM supplemented with $10 \%$ FBS. Two days after the treatment, cells were maintained in DMEM supplemented with $10 \%$ FBS and $1 \mu \mathrm{g} / \mathrm{ml}$ insulin for additional 5 days. The media was changed every 2 days.

\section{Oil Red O staining}

Cells were washed once with PBS, and subsequently fixed for 10 min in $4 \%$ formalin. After fixation, lipid droplets were stained by $5 \%$ Oil Red O in $60 \%$ isopropanol for $1 \mathrm{~h}$. Cells were washed twice with distilled water and observed using a microscope.

\section{Transfection of siRNAs, and infection of AVV}

Small interfering RNAs (siRNAs) of Senp2, Clebp $\beta$, Ppar $\gamma, \operatorname{Pgcl} \alpha$, and Prdm16 were purchased from Dharmacon (Chicago, IL, USA). Nonspecific siRNAs (siNS) were purchased from BIONEER (Daejeon, Korea). The siRNAs (100 nmol/l) were mixed with RNAiMAX (Invitrogen) in $100 \mu \mathrm{L}$ of serum-free DMEM and incubated for 30 min at room temperature. The complex was treated to cells with $400 \mu \mathrm{L}$ of serum-free DMEM. After $3 \mathrm{~h}$ incubation, $500 \mu \mathrm{l}$ of DMEM supplemented with $10 \%$ FBS was added. Adeno-associated virus (AAV)Flag-Hoxc10 was previously described (32). AAV-Flag-Hoxc10 was purified by AAV Mini Purification Kit (BioVision, Milpitas, CA, USA). Purified virus was diluted in Tris-HCl buffer at $1 / 100$ ratio and then treated to cells. 


\section{Plasmids and antibodies}

A DNA fragment from -1567 bp to -22 bp (from ATG) of the Hoxc10 promoter was inserted into the pGL2-basic vector (Promega, Madison, WI) to generate Hoxc10 (-1567)-Luc. In addition, Hoxc10 (-908)-Luc was constructed by inserting the promoter fragment from -908 bp to -22 bp of the mouse Hoxc10 promoter into pGL2-enhancer vector. To generate Hoxc10 (-1567 mt)-Luc, DNA sequences between -1034 bp and -1026 bp of the Hoxc10 (-1567)-Luc, 5'-TGGAGCAAAG-3', were changed to 5'- ACCTGCAAAG -3' by using the Quick-Change site directed mutagenesis Kit (Stratagene, La Jolla, CA, USA). Expression vectors for SENP2, C/EBP $\beta$ WT, C/EBP $\beta$ K133R, SUMO and UBC9 were described previously (33). The expression vector for FLAG-DAXX was a gift from Dr. C.H. Chung (Seoul National University). To generate SUMO-tagged C/EBP $\beta$, cDNA of SUMO1 was ligated between HA and $\mathrm{C} / \mathrm{EBP} \beta$ in pcDNA-HA-C/EBP $\beta W T$ or K133R. Antibodies against SENP2, HOXC10, DAXX, SMRT and GAPDH were purchased from Santa Cruz Biotechnology (Santa Cruz, CA, USA). Antibodies against $\mathrm{C} / \mathrm{EBP} \beta$ and UCP1 were obtained from Abcam (Cambridge, UK), and the $\gamma$-tubulin antibody and CL316243 were purchased from Sigma-Aldrich (St Louis, MO, USA).

\section{Transient transfection and luciferase reporter assay}

COS7 cells were cultured in DMEM supplemented with $10 \%$ FBS. COS7 cells were seeded at 12 -well plates and transfected with reporter vectors $(0.3 \mu \mathrm{g})$, expression vector for $\mathrm{C} / \mathrm{EBP} \beta$ 
WT, C/EBP $\beta$ K133R $(0.01 \mu \mathrm{g}$ or $0.025 \mu \mathrm{g})$, UBC9 $(0.1 \mu \mathrm{g})$, SUMO $(0.1 \mu \mathrm{g})$, SENP2 (0.01, 0.05 and $0.1 \mu \mathrm{g})$ and pRSV- $\beta$-galactosidase $(0.1 \mu \mathrm{g})$ using Lipofectamine and Plus reagent (Invitrogen). Luciferase and $\beta$-galactosidase activities were measured according to the manufacturer's instruction (Promega, Madison, WI).

\section{Immunoprecipitation and Western blot analysis}

For immunoprecipitation, COS7 cells plated on 6-well plates were transfected with expression vectors for HA-C/EBP $\beta$ WT or HA-C/EBP $\beta$ K133R (0.25 $\mu \mathrm{g})$, Flag-DAXX (1 $\mu \mathrm{g})$, Myc-SUMO $(0.5 \mu \mathrm{g})$ and Flag-UBC9 $(0.5 \mu \mathrm{g})$ by using Lipofectamin plus (Invitrogen). After incubation for $24 \mathrm{~h}$, cells were lysed in the RIPA buffer (Millipore) supplemented with protease inhibitors $(10 \mu \mathrm{g} / \mu \mathrm{l}$ aprotinin, $10 \mu \mathrm{g} / \mu \mathrm{l}$ leupeptin and $1 \mathrm{mM}$ PMSF). Cell lysates $(0.5 \mathrm{mg})$ were incubated with anti-HA antibody-coupled agarose beads (Roche, Basel, Switzerland) for $16 \mathrm{~h}$ at $4{ }^{\circ} \mathrm{C}$. The precipitates were washed five times and subjected to SDSpolyacrylamide gel electrophoresis. For western blot analysis of tissue or cell lysates, samples were prepared in RIPA buffer supplemented with protease inhibitors. Proteins on SDSpolyacrylamide gel were transferred onto a nitrocellulose membrane (GE Healthcare). The bands were visualized utilizing Enhanced Chemiluminescence (Pierce, Rockford, IL, USA).

\section{Chromatin IP linked qPCR}

SVF was isolated from iWAT of control or Senp2-aKO mice, and then differentiated to adipocytes for 7 days. After cross-linking and DNA fragmentation, nuclear extracts were immunoprecipitated with anti-C/EBP $\beta$, anti-DAXX, anti-SMRT or control IgG antibodies. 
Real-time qPCR was performed using the primers (Supplemental Table 1) for the C/EBP $\beta$ binding site $(-1030 \mathrm{bp})$ of the Hoxc10 promoter.

\section{Statistical analysis}

Statistical analysis was performed using SPSS version 12.0 (SPSS inc.). Statistical significances of the differences were determined by Student's $t$ test and 2-way ANOVA. $P<$ 0.05 was considered statistically significant.

\section{Acknowledgements}

This research was supported by Basic Science research Program through the National Research Foundation of Korea (NRF) funded by the Ministry of education (NRF2015R1D1A1A09059551) (NRF-2016R1A2B3010373) (NRF-2019R1A2C3009517) and by the National Institutes of Health (R01DK111529 to YBK).

\section{Author contributions}

K.S.P. and S.S.C. designed the study, interpreted the data and wrote the manuscript. J.S.L., J.N., Y.D.K., and S.L. performed the experiments and analyzed the results. S.C. and D.H. performed transcriptomic analysis. Y.B.K., W.H. and Y.J.P. interpreted the results and edited the manuscript.

\section{Declaration of interests}

The authors declare no competing interests. 


\section{References}

1. Sun, K., Kusminski, C.M., and Scherer, P.E. 2011. Adipose tissue remodeling and obesity. $J$ Clin Invest 121:2094-2101.

2. Cypess, A.M., Lehman, S., Williams, G., Tal, I., Rodman, D., Goldfine, A.B., Kuo, F.C., Palmer, E.L., Tseng, Y.H., Doria, A, et al. 2009. Identification and importance of brown adipose tissue in adult humans. NEng/ J Med 360:1509-1517.

3. Cypess, A.M., White, A.P., Vernochet, C., Schulz, T.J., Xue, R., Sass, C.A., Huang, T.L., Roberts-Toler, C., Weiner, L.S., Sze, C., et al. 2013. Anatomical localization, gene expression profiling and functional characterization of adult human neck brown fat. Nat Med 19:635-639.

4. Cannon, B., and Nedergaard, J. 2004. Brown adipose tissue: function and physiological significance. Physiol Rev 84:277-359.

5. Cousin, B., Cinti, S., Morroni, M., Raimbault, S., Ricquier, D., Penicaud, L., and Casteilla, L. 1992. Occurrence of brown adipocytes in rat white adipose tissue: molecular and morphological characterization. J Cell Sci103 ( Pt 4):931-942.

6. Wu, J., Bostrom, P., Sparks, L.M., Ye, L., Choi, J.H., Giang, A.H., Khandekar, M., Virtanen, K.A., Nuutila, P., Schaart, G., et al. 2012. Beige adipocytes are a distinct type of thermogenic fat cell in mouse and human. Cell 150:366-376.

7. Lidell, M.E., Betz, M.J., Dahlqvist Leinhard, O., Heglind, M., Elander, L., Slawik, M., Mussack, T., Nilsson, D., Romu, T., Nuutila, P., et al. 2013. Evidence for two types of brown adipose tissue in humans. Nat Med 19:631-634.

8. Barbatelli, G., Murano, I., Madsen, L., Hao, Q., Jimenez, M., Kristiansen, K., Giacobino, J.P., De Matteis, R., and Cinti, S. 2010. The emergence of cold-induced brown adipocytes in mouse white fat depots is determined predominantly by white to brown adipocyte transdifferentiation. Am J Physiol Endocrinol Metab 298:E1244-1253.

9. Wu, M.V., Bikopoulos, G., Hung, S., and Ceddia, R.B. 2014. Thermogenic capacity is antagonistically regulated in classical brown and white subcutaneous fat depots by high fat diet and endurance training in rats: impact on whole-body energy expenditure. $J$ Biol Chem 
289:34129-34140.

10. Park, J.W., Jung, K.H., Lee, J.H., Quach, C.H., Moon, S.H., Cho, Y.S., and Lee, K.H. 2015. 18F-FDG PET/CT monitoring of beta3 agonist-stimulated brown adipocyte recruitment in white adipose tissue. J Nucl Med 56:153-158.

11. Fukui, Y., Masui, S., Osada, S., Umesono, K., and Motojima, K. 2000. A new thiazolidinedione, NC-2100, which is a weak PPAR-gamma activator, exhibits potent antidiabetic effects and induces uncoupling protein 1 in white adipose tissue of KKAy obese mice. Diabetes 49:759-767.

12. Petrovic, N., Walden, T.B., Shabalina, I.G., Timmons, J.A., Cannon, B., and Nedergaard, J. 2010. Chronic peroxisome proliferator-activated receptor gamma (PPARgamma) activation of epididymally derived white adipocyte cultures reveals a population of thermogenically competent, UCP1-containing adipocytes molecularly distinct from classic brown adipocytes. $J$ Biol Chem 285:7153-7164.

13. Lin, J.Z., Martagon, A.J., Cimini, S.L., Gonzalez, D.D., Tinkey, D.W., Biter, A., Baxter, J.D., Webb, P., Gustafsson, J.A., Hartig, S.M., et al. 2015. Pharmacological Activation of Thyroid Hormone Receptors Elicits a Functional Conversion of White to Brown Fat. Cell Rep 13:15281537.

14. Lee, Y.H., Petkova, A.P., Konkar, A.A., and Granneman, J.G. 2015. Cellular origins of coldinduced brown adipocytes in adult mice. FASEB J29:286-299.

15. Rosenwald, M., Perdikari, A., Rulicke, T., and Wolfrum, C. 2013. Bi-directional interconversion of brite and white adipocytes. Nat Cell Biol 15:659-667.

16. Vishvanath, L., MacPherson, K.A., Hepler, C., Wang, Q.A., Shao, M., Spurgin, S.B., Wang, M.Y., Kusminski, C.M., Morley, T.S., and Gupta, R.K. 2016. Pdgfrbeta+ Mural Preadipocytes Contribute to Adipocyte Hyperplasia Induced by High-Fat-Diet Feeding and Prolonged Cold Exposure in Adult Mice. Cell Metab 23:350-359.

17. Long, J.Z., Svensson, K.J., Tsai, L., Zeng, X., Roh, H.C., Kong, X., Rao, R.R., Lou, J., Lokurkar, I., Baur, W., et al. 2014. A smooth muscle-like origin for beige adipocytes. Cell Metab 19:810-820.

18. Berry, D.C., Jiang, Y., and Graff, J.M. 2016. Mouse strains to study cold-inducible beige progenitors and beige adipocyte formation and function. Nat Commun 7:10184.

19. Wang, W., and Seale, P. 2016. Control of brown and beige fat development. Nat Rev Mol Cell Biol 17:691-702.

20. Pollard, A.E., and Carling, D. 2020. Thermogenic adipocytes: lineage, function and therapeutic potential. Biochem J477:2071-2093.

21. Yi, D., Nguyen, H.P., and Sul, H.S. 2020. Epigenetic dynamics of the thermogenic gene program of adipocytes. Biochem J477:1137-1148.

22. Seale, P. 2015. Transcriptional Regulatory Circuits Controlling Brown Fat Development and Activation. Diabetes 64:2369-2375.

23. Puigserver, P., Wu, Z., Park, C.W., Graves, R., Wright, M., and Spiegelman, B.M. 1998. A 
cold-inducible coactivator of nuclear receptors linked to adaptive thermogenesis. Cel/92:829839.

24. Kleiner, S., Mepani, R.J., Laznik, D., Ye, L., Jurczak, M.J., Jornayvaz, F.R., Estall, J.L., Chatterjee Bhowmick, D., Shulman, G.I., and Spiegelman, B.M. 2012. Development of insulin resistance in mice lacking PGC-1alpha in adipose tissues. Proc Natl Acad Sci U $S A$ 109:9635-9640.

25. Seale, P., Kajimura, S., Yang, W., Chin, S., Rohas, L.M., Uldry, M., Tavernier, G., Langin, D., and Spiegelman, B.M. 2007. Transcriptional control of brown fat determination by PRDM16. Cell Metab6:38-54.

26. Cohen, P., Levy, J.D., Zhang, Y., Frontini, A., Kolodin, D.P., Svensson, K.J., Lo, J.C., Zeng, X., Ye, L., Khandekar, M.J., et al. 2014. Ablation of PRDM16 and beige adipose causes metabolic dysfunction and a subcutaneous to visceral fat switch. Cell 156:304-316.

27. Seale, P., Conroe, H.M., Estall, J., Kajimura, S., Frontini, A., Ishibashi, J., Cohen, P., Cinti, S., and Spiegelman, B.M. 2011. Prdm 16 determines the thermogenic program of subcutaneous white adipose tissue in mice. J Clin Invest 121:96-105.

28. Gesta, S., Bluher, M., Yamamoto, Y., Norris, A.W., Berndt, J., Kralisch, S., Boucher, J., Lewis, C., and Kahn, C.R. 2006. Evidence for a role of developmental genes in the origin of obesity and body fat distribution. Proc Natl Acad Sci U S A 103:6676-6681.

29. Yamamoto, Y., Gesta, S., Lee, K.Y., Tran, T.T., Saadatirad, P., and Kahn, C.R. 2010. Adipose depots possess unique developmental gene signatures. Obesity (Silver Spring) 18:872-878.

30. Mori, M., Nakagami, H., Rodriguez-Araujo, G., Nimura, K., and Kaneda, Y. 2012. Essential role for miR-196a in brown adipogenesis of white fat progenitor cells. PLOS Biol 10:e1001314.

31. Brune, J.E., Kern, M., Kunath, A., Flehmig, G., Schon, M.R., Lohmann, T., Dressler, M., Dietrich, A., Fasshauer, M., Kovacs, P., et al. 2016. Fat depot-specific expression of HOXC9 and HOXC10 may contribute to adverse fat distribution and related metabolic traits. Obesity (Silver Spring) 24:51-59.

32. Ng, Y., Tan, S.X., Chia, S.Y., Tan, H.Y., Gun, S.Y., Sun, L., Hong, W., and Han, W. 2017. HOXC10 suppresses browning of white adipose tissues. Exp Mol Med 49:e292.

33. Chung, S.S., Ahn, B.Y., Kim, M., Choi, H.H., Park, H.S., Kang, S., Park, S.G., Kim, Y.B., Cho, Y.M., Lee, H.K., et al. 2010. Control of adipogenesis by the SUMO-specific protease SENP2. Mol Cell Bio/30:2135-2146.

34. Koo, Y.D., Choi, J.W., Kim, M., Chae, S., Ahn, B.Y., Kim, M., Oh, B.C., Hwang, D., Seol, J.H., Kim, Y.B., et al. 2015. SUMO-Specific Protease 2 (SENP2) Is an Important Regulator of Fatty Acid Metabolism in Skeletal Muscle. Diabetes 64:2420-2431.

35. Kim, J., Cantwell, C.A., Johnson, P.F., Pfarr, C.M., and Williams, S.C. 2002. Transcriptional activity of CCAAT/enhancer-binding proteins is controlled by a conserved inhibitory domain that is a target for sumoylation. $J$ Biol Chem 277:38037-38044.

36. Atwood, A.A., Jerrell, R., and Sealy, L. 2011. Negative regulation of C/EBPbeta1 by 
sumoylation in breast cancer cells. PLoS One 6:e25205.

37. Berberich-Siebelt, F., Berberich, I., Andrulis, M., Santner-Nanan, B., Jha, M.K., Klein-Hessling, S., Schimpl, A., and Serfling, E. 2006. SUMOylation interferes with CCAAT/enhancer-binding protein beta-mediated c-myc repression, but not IL-4 activation in T cells. $J$ Immunol 176:4843-4851.

38. Aguilar, R., Grandy, R., Meza, D., Sepulveda, H., Pihan, P., van Wijnen, A.J., Lian, J.B., Stein, G.S., Stein, J.L., and Montecino, M. 2014. A functional N-terminal domain in C/EBPbeta-LAP* is required for interacting with SWI/SNF and to repress Ric-8B gene transcription in osteoblasts. J Cell Physio/229:1521-1528.

39. Raghav, S.K., Waszak, S.M., Krier, I., Gubelmann, C., Isakova, A., Mikkelsen, T.S., and Deplancke, B. 2012. Integrative genomics identifies the corepressor SMRT as a gatekeeper of adipogenesis through the transcription factors C/EBPbeta and KAISO. Mol Cel/46:335-350.

40. Escobar-Cabrera, E., Okon, M., Lau, D.K., Dart, C.F., Bonvin, A.M., and McIntosh, L.P. 2011. Characterizing the $\mathrm{N}$ - and $\mathrm{C}$-terminal Small ubiquitin-like modifier (SUMO)-interacting motifs of the scaffold protein DAXX. J Biol Chem 286:19816-19829.

41. Lin, D.Y., Huang, Y.S., Jeng, J.C., Kuo, H.Y., Chang, C.C., Chao, T.T., Ho, C.C., Chen, Y.C., Lin, T.P., Fang, H.I., et al. 2006. Role of SUMO-interacting motif in Daxx SUMO modification, subnuclear localization, and repression of sumoylated transcription factors. $\mathrm{Mol}$ Cel/ 24:341354.

42. Kuo, H.Y., Chang, C.C., Jeng, J.C., Hu, H.M., Lin, D.Y., Maul, G.G., Kwok, R.P., and Shih, H.M. 2005. SUMO modification negatively modulates the transcriptional activity of CREBbinding protein via the recruitment of Daxx. Proc Natl Acad Sci U S A 102:16973-16978.

43. Kaltenecker, D., Spirk, K., Ruge, F., Grebien, F., Herling, M., Rupprecht, A., Kenner, L., Pohl, E.E., Mueller, K.M., and Moriggl, R. 2020. STAT5 is required for lipid breakdown and betaadrenergic responsiveness of brown adipose tissue. Mol Metab 40:101026.

44. Wei, S., Zhang, M., Zheng, Y., and Yan, P. 2018. ZBTB16 Overexpression Enhances White Adipogenesis and Induces Brown-Like Adipocyte Formation of Bovine White Intramuscular Preadipocytes. Cell Physiol Biochem 48:2528-2538.

45. Nueda, M.L., Gonzalez-Gomez, M.J., Rodriguez-Cano, M.M., Monsalve, E.M., Diaz-Guerra, M.J.M., Sanchez-Solana, B., Laborda, J., and Baladron, V. 2018. DLK proteins modulate NOTCH signaling to influence a brown or white 3T3-L1 adipocyte fate. Sci Rep 8:16923.

46. Tan, C.K., Chong, H.C., Tan, E.H., and Tan, N.S. 2012. Getting 'Smad' about obesity and diabetes. Nutr Diabetes 2:e29.

47. Zhu, Q., Chang, A., Xu, A., and Luo, K. 2018. The regulatory protein SnoN antagonizes activin/Smad2 protein signaling and thereby promotes adipocyte differentiation and obesity in mice. J Biol Chem 293:14100-14111.

48. McFie, P.J., Wang, G.L., Timchenko, N.A., Wilson, H.L., Hu, X., and Roesler, W.J. 2006. Identification of a co-repressor that inhibits the transcriptional and growth-arrest activities of CCAAT/enhancer-binding protein alpha. J Biol Chem 281:18069-18080. 
49. !!! INVALID CITATION !!!

50. Burl, R.B., Ramseyer, V.D., Rondini, E.A., Pique-Regi, R., Lee, Y.H., and Granneman, J.G. 2018. Deconstructing Adipogenesis Induced by beta3-Adrenergic Receptor Activation with Single-Cell Expression Profiling. Cell Metab 28:300-309 e304.

51. Gao, Z., Daquinag, A.C., Su, F., Snyder, B., and Kolonin, M.G. 2018. PDGFRalpha/PDGFRbeta signaling balance modulates progenitor cell differentiation into white and beige adipocytes. Development 145.

52. Carmona, M.C., Hondares, E., Rodriguez de la Concepcion, M.L., Rodriguez-Sureda, V., Peinado-Onsurbe, J., Poli, V., Iglesias, R., Villarroya, F., and Giralt, M. 2005. Defective thermoregulation, impaired lipid metabolism, but preserved adrenergic induction of gene expression in brown fat of mice lacking C/EBPbeta. Biochem J389:47-56.

53. Zheng, Q., Cao, Y., Chen, Y., Wang, J., Fan, Q., Huang, X., Wang, Y., Wang, T., Wang, X., Ma, J., et al. 2018. Senp2 regulates adipose lipid storage by de-SUMOylation of Setdb1. J Mol Cell Biol 10:258-266.

54. Liang, Q., Zheng, Q., Zuo, Y., Chen, Y., Ma, J., Ni, P., and Cheng, J. 2019. SENP2 Suppresses Necdin Expression to Promote Brown Adipocyte Differentiation. Cell Rep 28:2004-2011 e2004.

55. Koo, Y.D., Lee, J.S., Lee, S.A., Quaresma, P.G.F., Bhat, R., Haynes, W.G., Park, Y.J., Kim, Y.B., Chung, S.S., and Park, K.S. 2019. SUMO-specific protease 2 mediates leptin-induced fatty acid oxidation in skeletal muscle. Metabolism 95:27-35.

56. Bolstad, B.M., Irizarry, R.A., Astrand, M., and Speed, T.P. 2003. A comparison of normalization methods for high density oligonucleotide array data based on variance and bias. Bioinformatics 19:185-193.

57. Chae, S., Ahn, B.Y., Byun, K., Cho, Y.M., Yu, M.H., Lee, B., Hwang, D., and Park, K.S. 2013. A systems approach for decoding mitochondrial retrograde signaling pathways. Sci Signal 6:rs4.

58. Hwang, D., Rust, A.G., Ramsey, S., Smith, J.J., Leslie, D.M., Weston, A.D., de Atauri, P., Aitchison, J.D., Hood, L., Siegel, A.F., et al. 2005. A data integration methodology for systems biology. Proc Natl Acad SciUS A 102:17296-17301.

59. Huang da, W., Sherman, B.T., and Lempicki, R.A. 2009. Systematic and integrative analysis of large gene lists using DAVID bioinformatics resources. Nat Protoc 4:44-57.

60. Trapnell, C., Pachter, L., and Salzberg, S.L. 2009. TopHat: discovering splice junctions with RNA-Seq. Bioinformatics 25:1105-1111.

61. Anders, S., Pyl, P.T., and Huber, W. 2015. HTSeq--a Python framework to work with highthroughput sequencing data. Bioinfomatics 31:166-169.

62. Robinson, M.D., and Oshlack, A. 2010. A scaling normalization method for differential expression analysis of RNA-seq data. Genome Biol11:R25.

63. Kim, Y., Kim, T.K., Kim, Y., Yoo, J., You, S., Lee, I., Carlson, G., Hood, L., Choi, S., and Hwang, D. 2011. Principal network analysis: identification of subnetworks representing major 
bioRxiv preprint doi: https://doi.org/10.1101/2020.12.16.422969; this version posted December 17, 2020. The copyright holder for this preprint (which was not certified by peer review) is the author/funder. All rights reserved. No reuse allowed without permission.

dynamics using gene expression data. Bioinformatics 27:391-398. 


\section{Figure legends}

Figure 1. Accumulation of beige adipocytes in iWAT of Senp2-aKO mice (A) Generation of Senp2-aKO mice. (B) Expression of SENP2 in adipose tissues of Senp2-floxed mice (control) and Senp2-aKO mice (aKO). (C-L) Control and Senp2-aKO mice were fed a normal chow diet (NCD) for 16 weeks. (C) Body weights of 16 week-old control and Senp2-aKO mice. $\mathrm{n}=12-15$. (D) Each fat pad weight was expressed as $\%$ of each body weight. $* P<0.05$ by Student's t-test, $n=11-15$. (E) Glucose tolerance test (GTT) and (F) insulin tolerance test (ITT) $* P<0.05$ by Student's t-test, $\mathrm{n}=10-12$. (G) Representative H\&E staining of eWAT, iWAT, and BAT from control and Senp2-aKO mice. Scale bar, $100 \mu \mathrm{m} .(\mathrm{H})$ Average size of adipocytes in eWAT and iWAT. $* P<0.05$ by Student's t-test, $\mathrm{n}=4-5$. (I) Immunohistochemical staining of iWAT using an UCP1 antibody. Scale bar, $100 \mu \mathrm{m}$. (J) Western blot analysis using an UCP1 antibody. (K) Control and Senp2-aKO mice (16-17-week old) were exposed to cold temperature $\left(4^{\circ} \mathrm{C}\right)$ for $3 \mathrm{~h}$. Body temperature was measured every $30 \mathrm{~min} . * P$ $<0.05$ by Student's t-test, $\mathrm{n}=10-12$. All data are presented as mean \pm SEM.

Figure 2. Adipocyte-specific depletion of SENP2 ameliorates HFD-induced obesity and insulin resistance. (A) Body weights of control and Senp2-aKO mice were measured during 12 weeks of HFD feeding. $* P<0.05$ by Student's t-test, $\mathrm{n}=19$. (B) Fat mass and lean mass were measured using DEXA after 14 weeks of HFD feeding (left and middle panels), and \% fat weight/body weight was also shown (right panel). ${ }^{*} P<0.05$ by Student's t-test, $\mathrm{n}=9-11$ (C) Fat pad weight of control and Senp2-aKO mice was measured after 14 weeks of HFD 
feeding. $* P<0.05$ by Student's t-test, $\mathrm{n}=8-11$. (D) GTT and (E) ITT after 12 or 13 weeks of HFD feeding, respectively. ${ }^{*} P<0.05$ by Student's t-test, $\mathrm{n}=13-15$. (F) Representative H\&E staining of WATs, BAT and liver tissues after 14 weeks of HFD feeding. The right panel shows enlarged photos of iWAT. Scale bar, $100 \mu \mathrm{m}$. (G) Average size of adipocytes in eWAT and iWAT. $* P<0.05$ by Student's t-test, $\mathrm{n}=4-5$. $(\mathrm{H})$ Volume oxygen $\left(\mathrm{O}_{2}\right)$ consumption rates and $(\mathrm{J})$ heat production rates at week 15 of HFD-feeding. ( $\mathrm{I}$ and $\mathrm{K}$ ) Average $\mathrm{O}_{2}$ consumption and average heat production of 3 day measurements. $* P<0.05$ by Student's t-test, $\mathrm{n}=10-12$. All data are presented as mean \pm SEM.

Figure 3. SENP2 depletion induced thermogenic gene expression in iWAT (A) Volcano plot (left) showing up- (red) and down-regulated (green) genes in iWAT from Senp2-aKO mice, compared to that from control mice on a HFD for 14 weeks. Cellular processes enriched by the up- (top) and down-regulated (bottom) genes are also shown in the bar plots (right) where the enrichment significance (p-value) was displayed as $-\log _{10}(\mathrm{p})$. (B) Heat map showing the $\log _{2}$-fold changes of the indicated genes with respect to their median mRNA expression levels in control and Senp2-aKO iWAT. $\mathrm{n}=4$. (C) Relative gene expression in iWAT of HFD-fed mice was determined by qPCR. The mRNA level of each gene in the control mice were set to $1 .{ }^{*} P<0.05$ by Student's t-test, $\mathrm{n}=14-15$. (D) Relative gene expression in iWAT of NCD-fed mice. $* P<0.05$ by Student's t-test, $\mathrm{n}=13-14$. All data are presented as mean \pm SEM.

Figure 4. SENP2 depletion facilitates differentiation of white adipose progenitor cells 
toward thermogenic adipocytes (A-C) SVF was isolated from iWAT of control and Senp2aKO mice and then differentiated to adipocytes. (B) Oil-Red $\mathrm{O}$ staining at day 7 of differentiation. (C) Relative mRNA levels of BAT specific genes and some transcription factors at day 7 of differentiation. The values obtained in the cells from control mice were set to1. ${ }^{*} P<0.05$ by Student's t-test, $\mathrm{n}=9-10$. (D-E) At day 7 of differentiation, CL31624 (10 $\mu \mathrm{mol} / \mathrm{L}$ ) were treated for $5 \mathrm{~h}$. The mRNA levels were measured (D), and the values in the cells from control mice without CL31624 treatment were set to 1 and the others were its relative values. ${ }^{*} P<0.05$ vs control/without CL31624 by Student's t-test, ${ }^{\#} P<0.05$ vs aKO/without CL31624 by 2-way Anova. $n=4$. (E) Western blotting was performed. (F) At different points of time $(0,2,4$ and 7 day) after the induction of adipocyte differentiation from SVF, RNAs were isolated and subjected to qPCR. The values of control cells at day 0 were set to 1 and the others were its relative values. $* P<0.05$ vs control by 2 -way Anova, $\mathrm{n}$ $=3$. All data are represented as the mean \pm SEM.

Figure 5. Suppression of HOXC10 stimulates differentiation of SENP2-deficient adipocytes toward beige adipocytes. (A-C) SVF isolated from iWAT of control or Senp2aKO mice were differentiated to adipocytes for 5 days, and then infected with AAV-Hoxc10 for 2 days. Cells were harvested and western blotting (B) and qPCR (C) were performed. The values of cells from control mice without AAV-Hoxc10 treatment were set to 1 , and the others were expressed as its relative values. ${ }^{*} P<0.05$ vs control without AAV-Hoxc10 by Student's t-test, ${ }^{\#} P<0.05$ vs aKO without AAV-Hoxc10 by 2 -way Anova, $\mathrm{n}=4$. (D-I) Cells were treated with siRNAs targeting the indicated transcription factors or siNS (non-specific) at day 5 of differentiation. Relative mRNA levels of Ucpl (E), Cidea (F), Elovl3 (G) and Hoxc10 (H) were determined. The values of cells from control mice treated siNS were set to 1 and the 
others were expressed as its relative values. ${ }^{*} P<0.05$ vs control/siNS by Student's t-test, ${ }^{\#} P$ $<0.05$ vs aKO/siNS by 2-way Anova, $\mathrm{n}=4-5$. (I) Western blotting was performed after siC/ebp $\beta$ treatment. All data are represented as the mean \pm SEM.

Figure 6. Inhibition of Hoxc10 transcription by sumoylated C/EBPß. (A) Cos 7 cells were transfected with Hoxc10 (-1567 bp)-Luc, expression vectors for C/EBP $\beta$ WT or K133R, UBC9, SUMO and $\beta$-galactosidase. Luciferase activity of the cells transfected with Hoxc10 ($1567 \mathrm{bp}$ )-Luc without C/EBP $\beta$ was set to 100 and the others were expressed as its relative values. ${ }^{*} P<0.05$ vs without $\mathrm{C} / \mathrm{EBP} \beta$ by Student's t-test, $\mathrm{n}=4$. (B-C) Cells were transfected with different amounts of SENP2 expression vector. Luciferase activity (B) was measured. ${ }^{*} P<0.05$ vs without $\mathrm{C} / \mathrm{EBP} \beta,{ }^{\#} P<0.05$ vs $\mathrm{C} / \mathrm{EBP} \beta$ WT without SENP2 by 2 -way Anova. $\mathrm{n}$ $=3$. Western blotting $(\mathrm{C})$ was performed. Longer exposure of the anti-C/EBP $\beta$ blot shows sumoylated form of C/EBP $\beta$ (Top panel). (D-E) After transfection of C/EBP $\beta$ expression vectors and Hoxc10-Luc, western blot analysis (D) and luciferase assays (E) were performed. $* P<0.05$ vs without $\mathrm{C} / \mathrm{EBP} \beta$ by Student's t-test, $\mathrm{n}=4$. (F) Cells were transfected with different Hoxc10-Luc constructs. $* P<0.05$ vs without C/EBP $\beta W T$ by Student's t-test, $\mathrm{n}=5$ (upper panel), $\mathrm{n}=3$ (lower panel). (G) Adipocytes differentiated from iWAT SVF of control or Senp2-aKO mice were subjected for ChIP using an antibody of C/EBP $\beta$, DAXX or SMRT. $* P<0.05$ vs cells from control mice by Student's t-test, $\mathrm{n}=5$. $(\mathrm{H})$ Cos 7 cells were transfected with the indicated expression vectors, and then immunoprecipitation was performed with an anti-HA antibody. All data are represented as the mean \pm SEM. (I) A model showing the regulation of Hoxc10 transcription by SENP2. All data are represented as the mean \pm SEM. 
Figure 7. SENP2 depletion-induced thermogenic adipocyte differentiation through C/EBPß in both iWAT and eWAT. (A-B) SVF isolated from iWAT of control mice was differentiated to adipocytes, and treated with siRNAs at day 3 or day 7 of differentiation. (B) qPCR was performed four days after siRNA treatment. The values of siNS-treated cells were set to $1 .{ }^{*} P<0.05$ vs siNS by Student's t-test, $\mathrm{n}=5$. (C) SVF cells, treated with siNS or siSenp2 at day 3, were infected with AAV-Hoxc10 at day 5 of differentiation. Two days after virus infection, cells were harvested and qPCR was performed. The values of siNS-treated cells without AAV-Hoxc10 were set to $1 .{ }^{*} P<0.05$ vs siNS without AAV-Hoxc10, ${ }^{\#} P<0.05$ vs siSENP2 without AAV-Hoxc10 by 2 -way Anova, $\mathrm{n}=5$. (D-E) SVF was isolated from eWAT of control mice and treated with siRNAs. , $n=4$. (F-G) eWAT SVF from control and Senp2-aKO mice was treated with the indicated siRNAs. eWAT SVF of control and Senp2aKO mice were treated with siRNAs. The values of control mice/siNS treated cells were set to $1 .{ }^{*} P<0.05$ vs siNS/control, ${ }^{\#} P<0.05$ vs siNS/aKO by 2 -way Anova, $\mathrm{n}=4$. All data are represented as the mean \pm SEM. (H) Heat maps show the mean $\log _{2}$-fold changes of mRNA expression levels in the indicated conditions. (I) A model showing a function of SENP2 during differentiation of white adipose progenitor cells.

\section{Supplemental Figures}

\section{Supplemental Figure 1. Generation of adipocyte-specific Senp2 knockout mice (A)}

SENP2 protein levels in several tissues, gastronecmius (GM), liver and WATs, were compared by western blot analysis. (B) Senp2 mRNA levels in adipose tissues were measured by qPCR. The values of control mice were set to1. ${ }^{*} P<0.05$ vs control by Student's t-test, $\mathrm{n}$ 
=13-14. (C) After fractionation of adipocytes and stromal vascular fraction (SVF) from WAT, the lysates were subjected to western blotting of SENP2. (D) Representative western blots showing SENP2 protein levels in gastronecmius muscle (GM) and liver.

Supplemental Figure 2. Tissue mass and serum parameters of control and Senp2-aKO mice on a HFD (A) Average food intake during HFD feeding. $n=5-7$. (B) After 14 weeks of HFD feeding, bone mass density (BMD) and bone mass were measured. $n=9-11$. (C) Weight of liver and GM tissues. $n=9-11$. (D) Serum insulin, (E) serum triacylglycerol (TG) and free fatty acids (FFA) after HFD feeding. $\mathrm{n}=16-22$. (F) Serum leptin and adiponectin levels. $* P<$ 0.05 by Student's t-test, $\mathrm{n}=21-22$. All data are presented as mean \pm SEM.

Supplemental Figure 3. Gene expression profiles in iWAT and eWAT of control and Senp2-aKO mice. (A) Heat map showing the mRNA levels of transcription factors in iWAT of HFD-fed mice. $n=4$. (B-C) Relative mRNA levels of the indicated genes in eWAT of mice on a NCD (B) or HFD (C). The values obtained from control mice were set to $1 .{ }^{*} P<0.05$ by Student's t-test, $\mathrm{n}=13-15$. (D) Relative mRNAs of Senp2, C/ebpb and Hoxc10 in adipose tissues $n=3$. All data are presented as mean \pm SEM.

\section{Supplemental Figure 4. Expression of thermogenic genes during differentiation of SENP2-defiecent cells Relative mRNA levels of Cidea, Elovl3 and Pgcla. The values of control cells at day 0 were set to 1 and the others were its relative values. ${ }^{*} P<0.05$ vs control by 2 -way Anova, $\mathrm{n}=3$. All data are represented as the mean $\pm \mathrm{SEM}$.}




\section{Supplemental Figure 5. Knockdown of several transcription factors during} differentiation of adipose progenitor cells (A) Hoxcl0 mRNA levels were determined 2 days after AAV-Hoxc10 infection. The values of the control cells not treated with AAVHoxc10 were set to1. ${ }^{*} P<0.05$ vs control without AAV-Hoxc10 by Student's t-test, ${ }^{\#} P<0.05$ vs aKO without AAV-Hoxc10 by 2 -way Anova, n = 4. (B) Knockdown of Ppar $\gamma$ and Clebp $\beta$ was confirmed. (C) The mRNA levels of Senp2, Pgcla and Prdm16 were analyzed after knockdown of the transcription factors. The value of the control cells treated with siNS was set to1. ${ }^{*} P<0.05$ vs control/siNS by Student's t-test, ${ }^{\#} P<0.05$ vs aKO/siNS by 2 -way Anova, $\mathrm{n}=4-5$. All data are represented as the mean \pm SEM.

Supplemental Figure 6. Expression of C/EBP $\boldsymbol{\beta}$ after transfection Cos7 cells were transfected with C/EBP $\beta$ expression vectors $(5,10$ and $25 \mathrm{ng})$, pcDNA-HA-C/EBP $\beta W T$ and pcDNA-HA-C/EBP $\beta$ K133R. Expression of C/EBP $\beta$ was confirmed by western blot analysis.

Supplemental Figure 7. Effects of overexpression of Hoxc10 on beige adipogenesis of SENP2-deficient progenitor cells (A) iWAT SVF cells, treated with siNS or siSenp2 at day 3, were infected with AAV-Hoxc10 at day 5 of differentiation. The values of siNS-treated cells without AAV-Hoxc10 were set to $1 .{ }^{*} P<0.05$ vs siNS without AAV-Hoxc10, ${ }^{\#} P<0.05$ vs siSENP2 without AAV-Hoxc10 by 2-way Anova, $\mathrm{n}=5$. (B) eWAT SVF were treated with siRNAs. The values of control /siNS treated cells were set to $1 .{ }^{*} P<0.05$ vs siNS/control, ${ }^{\#} P$ $<0.05$ vs siNS/aKO by 2 -way Anova, $\mathrm{n}=4$. All data are represented as the mean \pm SEM. 


\section{Supplemental Figure 8. Profiles of genes showing C/EBP $\beta$-dependent expression} changes in both eWAT and iWAT of Senp2-aKO (A) Heat maps with respect to the mean expression levels in control (Cont_siNS), for genes in the clusters showing C/EBP $\beta$-mediated up-regulation (A; Clusters 6, 16, 20, 30) and down-regulation (B; Clusters 19, 21, 22, 25) in eWAT and iWAT. Cellular processes enriched by the up- (Group A) and down-regulated (Group B) genes are also shown in the bar plots and the enrichment significance (p-value) was displayed as $-\log _{10}(\mathrm{p})$. (B) Hoxc10 was overexpressed by infection of AAV-Hoxc10 in iWAT SVF-derived adipocytes, and qPCR was performed. The values of cells from control mice without AAV-Hoxc10 treatment were set to 1, and the others were expressed as its relative values. ${ }^{*} P<0.05$ vs control without AAV-Hoxc10 by Student's t-test, ${ }^{\#} P<0.05$ vs aKO without AAV-Hoxc10 by 2-way Anova, $\mathrm{n}=4$. 


\section{Figure1}

A

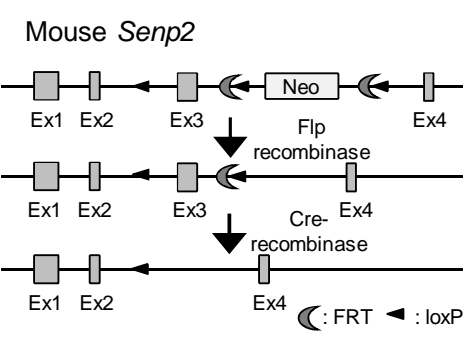

B

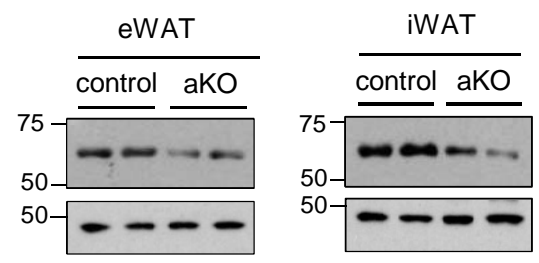

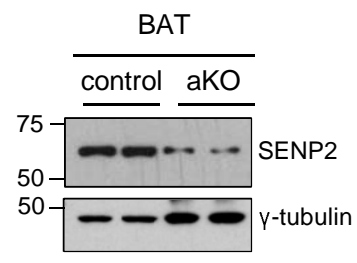

E
C

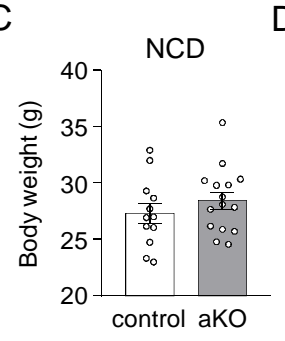

F

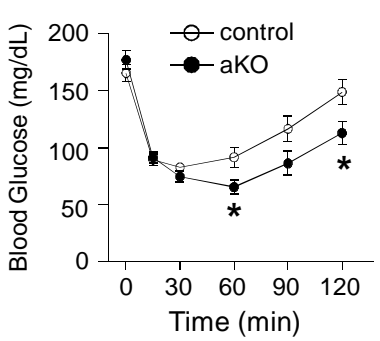

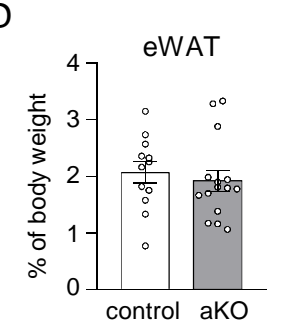
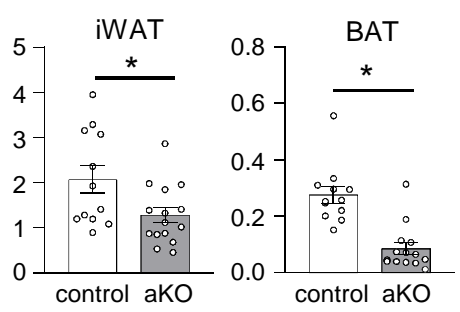

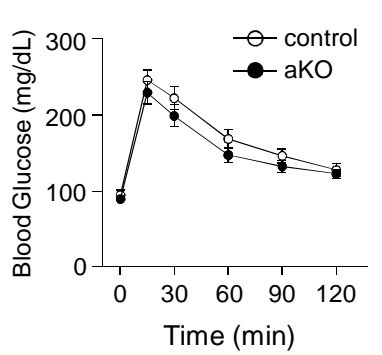

$\mathrm{H}$

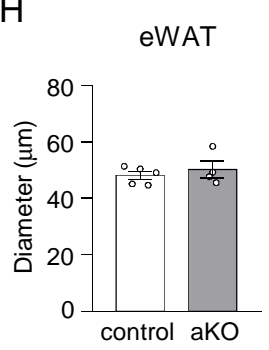

G
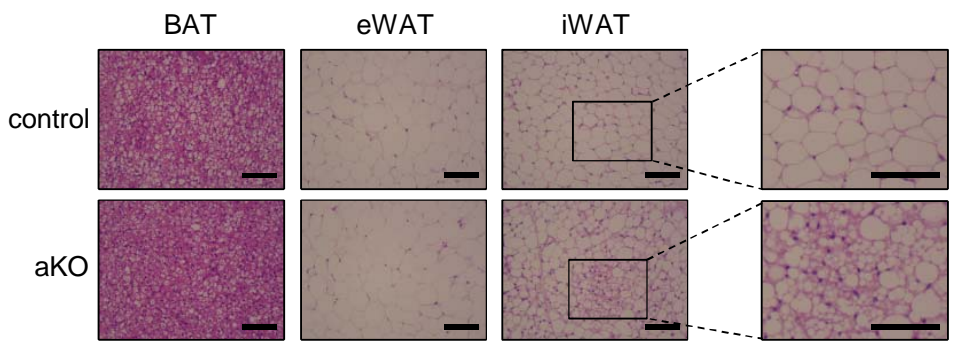

K

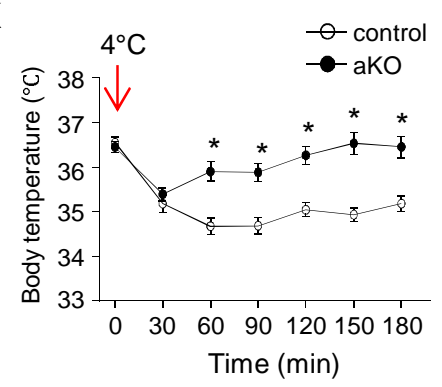




\section{Figure 2}

A

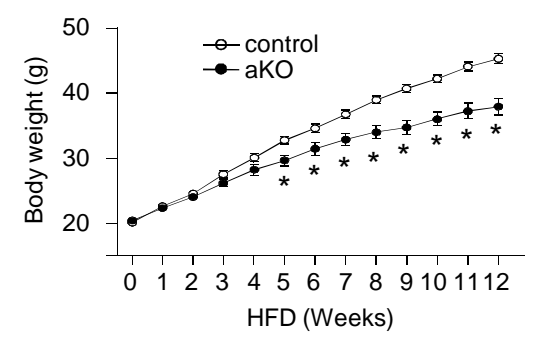

C

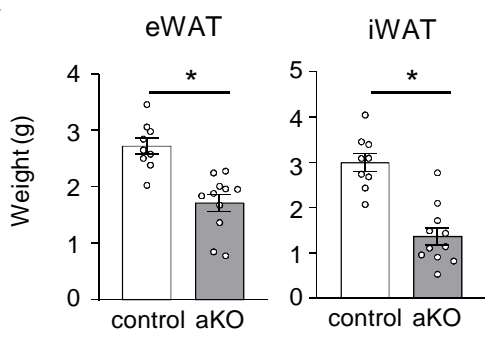

B
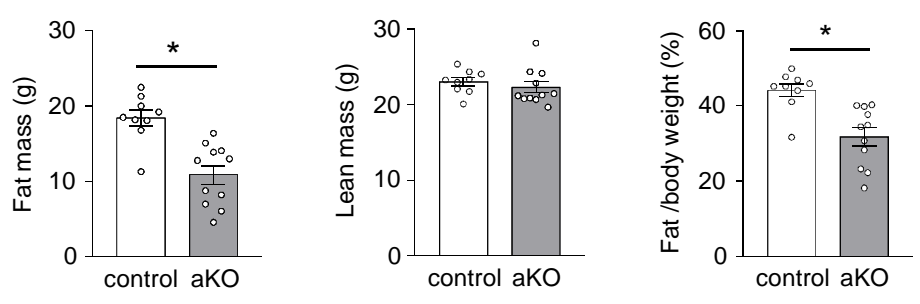

E

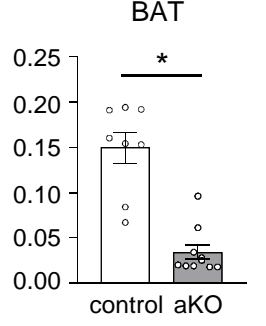

D

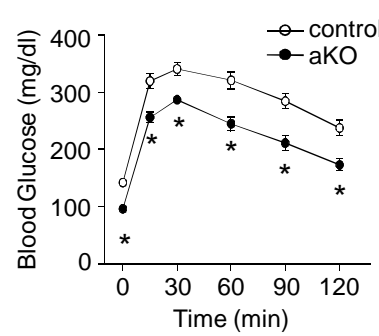

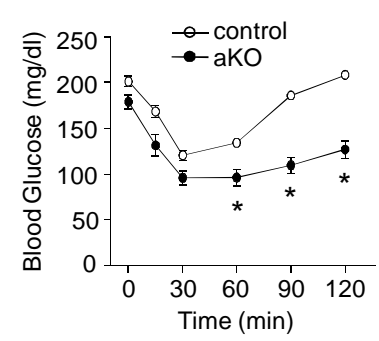

$\mathrm{F}$

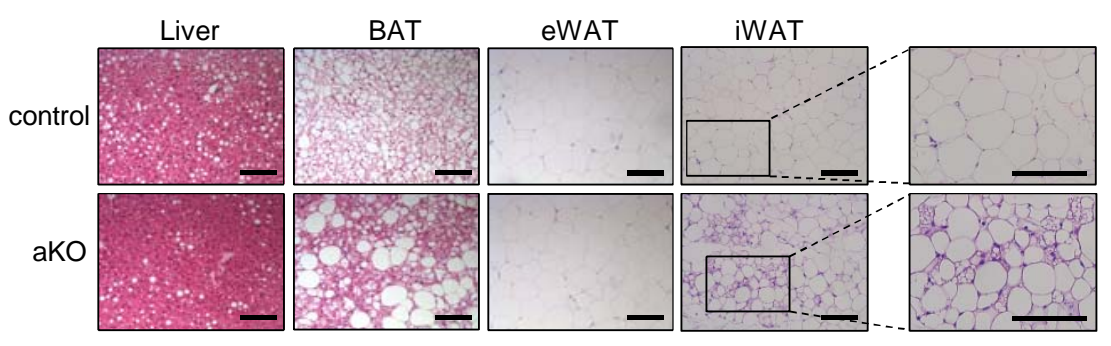

G

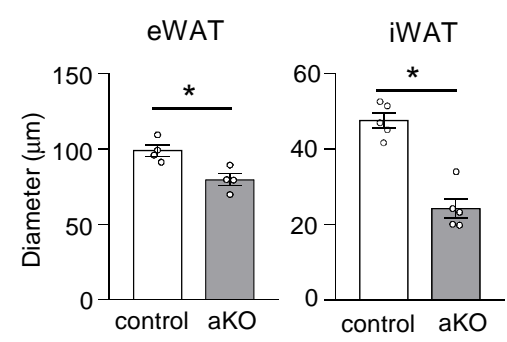

$\mathrm{H}$

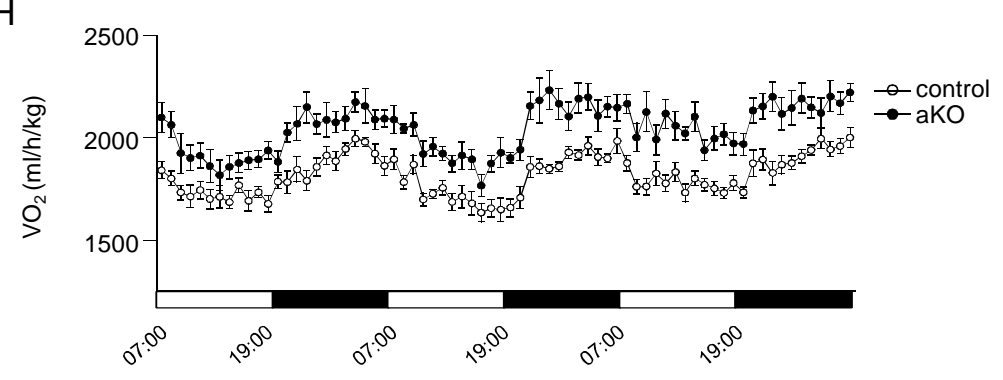

J

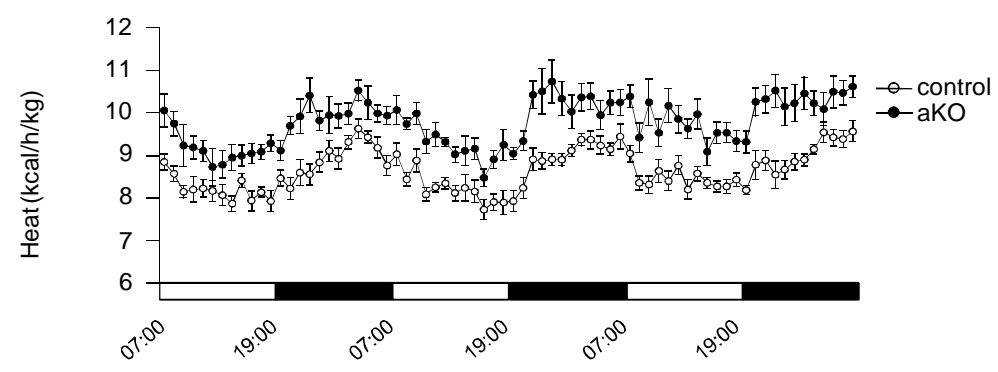

I

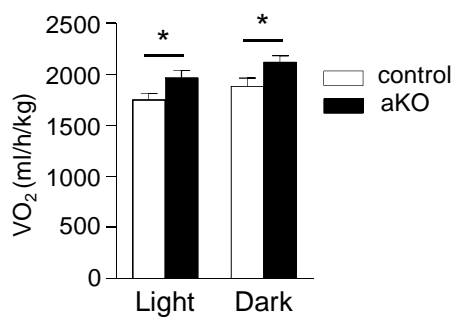

K

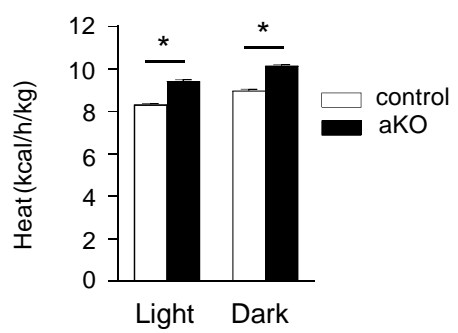




\section{Figure 3}

A

iWAT, HFD

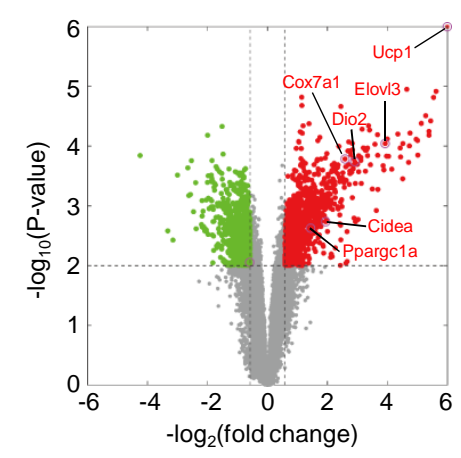

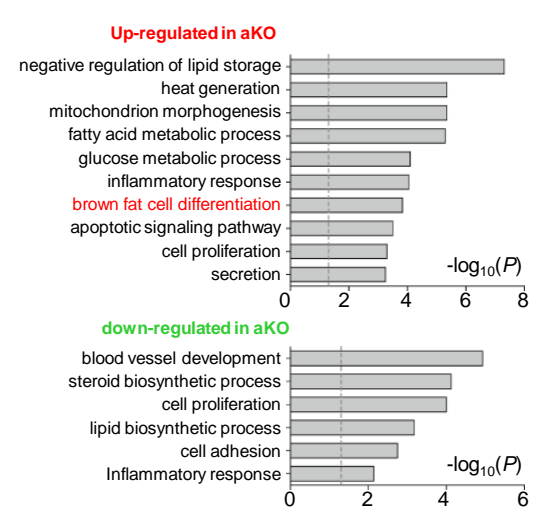

B

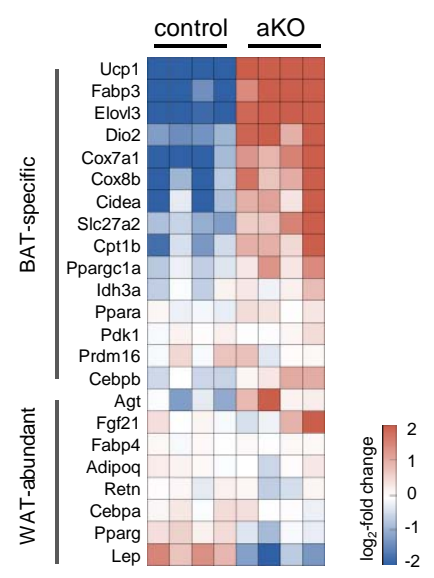

C
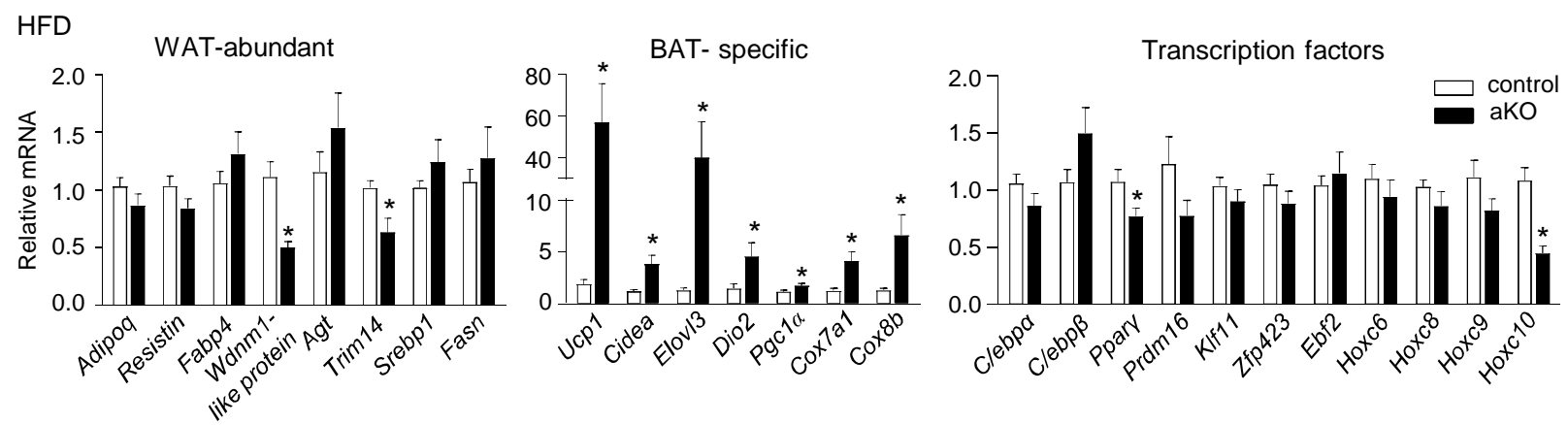

D
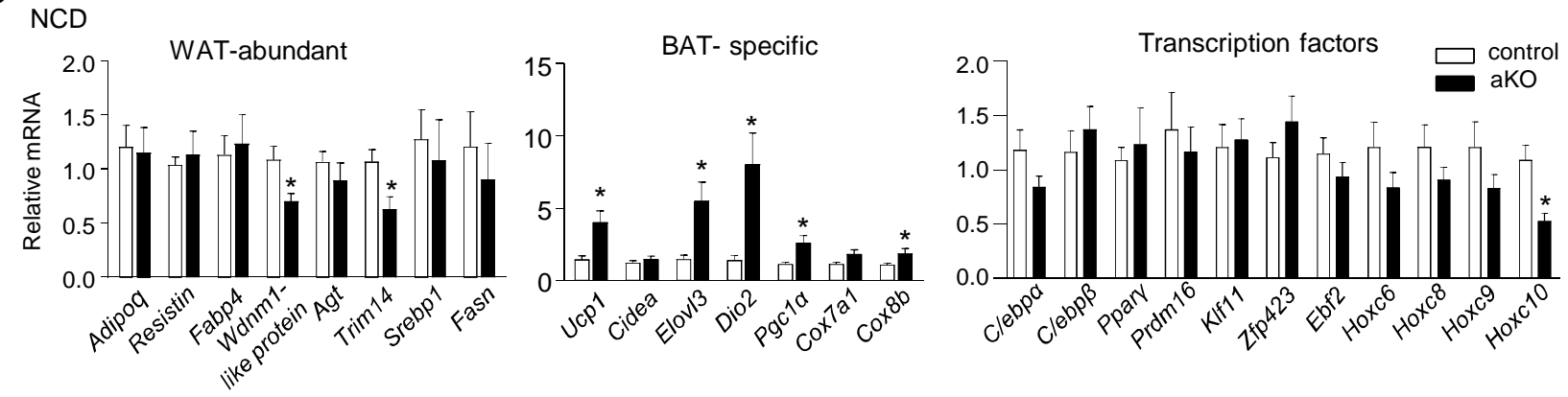


\section{Figure 4}

A

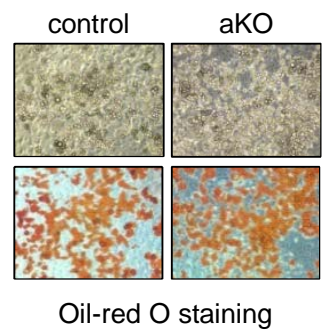

D

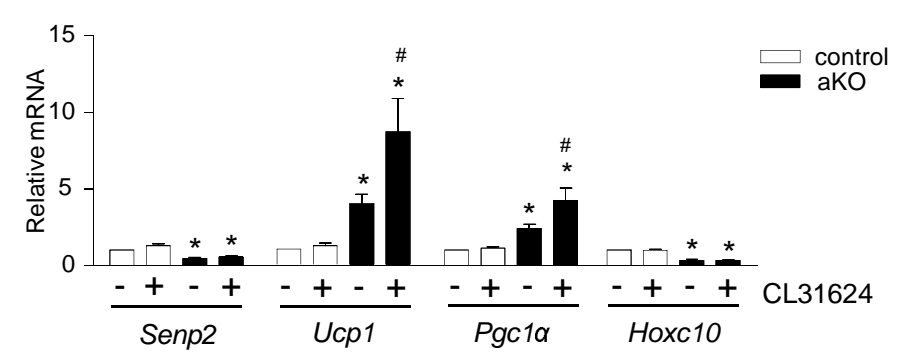

F

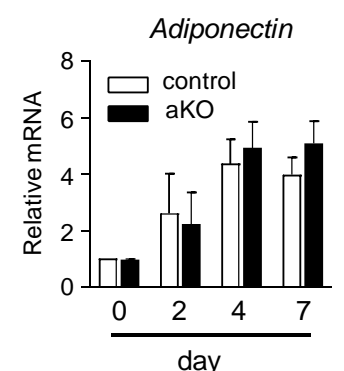

Adipocytes

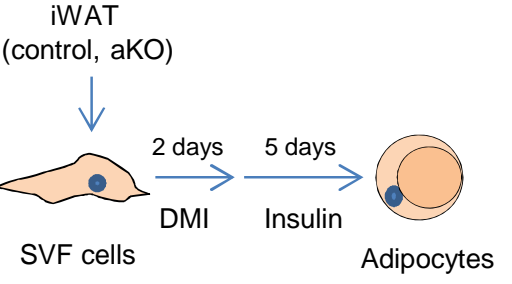

SVF cells
B

Oil-red O staining

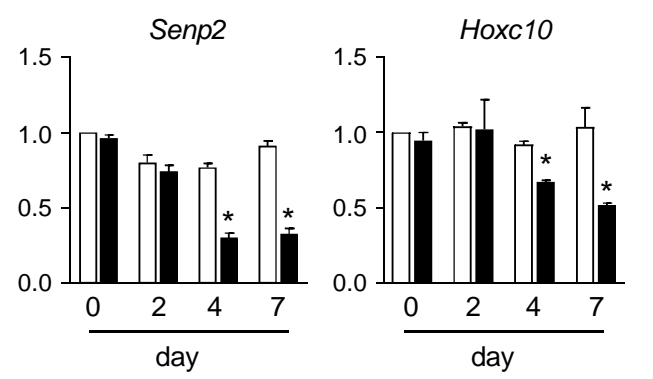

C

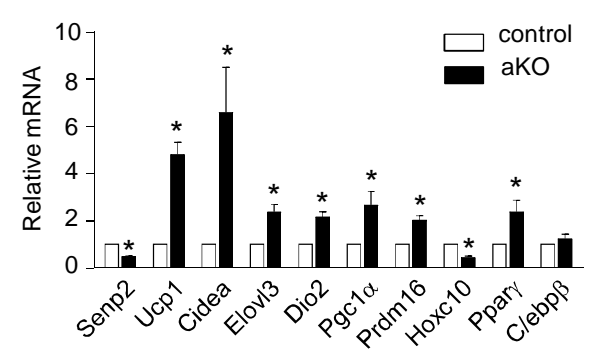

E
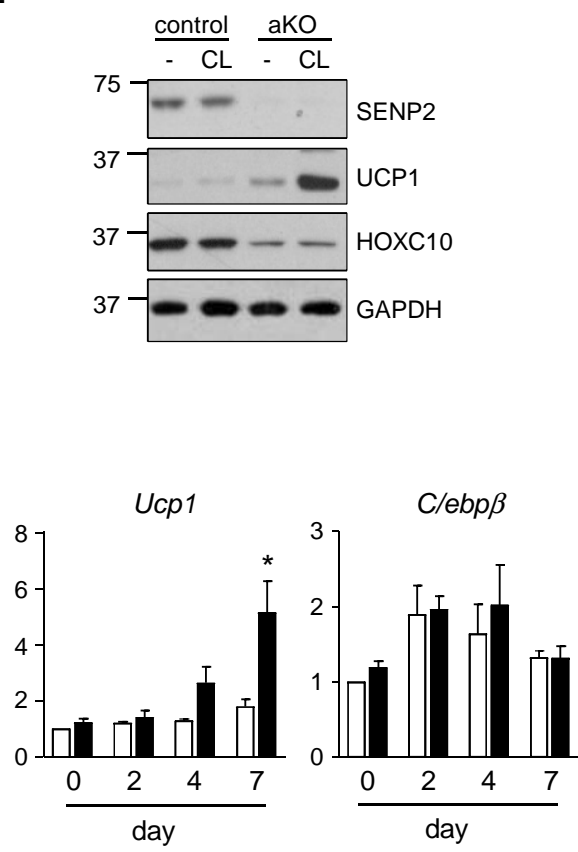


\section{Figure 5}

A

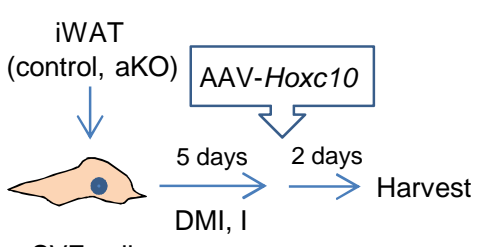

SVF cells
B

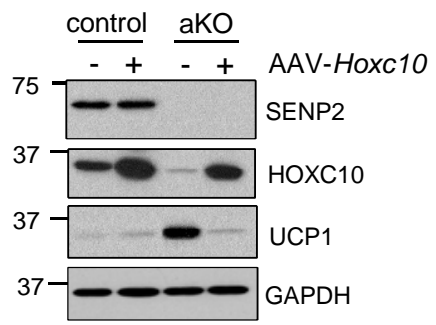

C

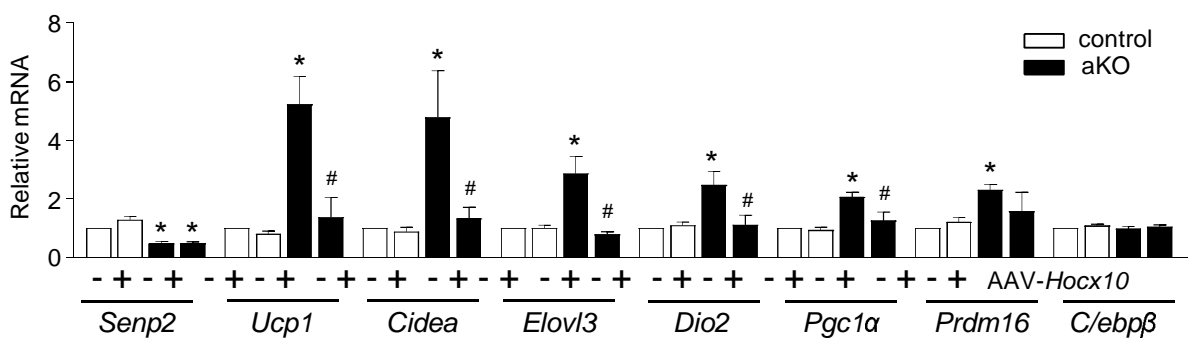

D

iWAT

(control, aKO)

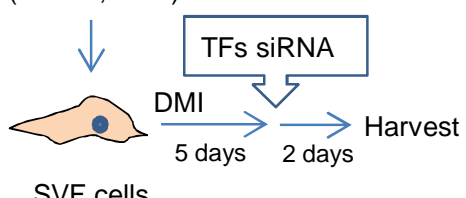

SVF cells

G

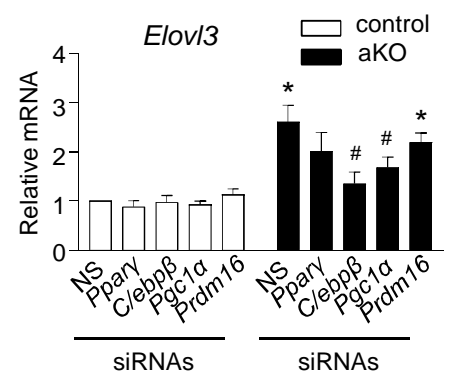

E

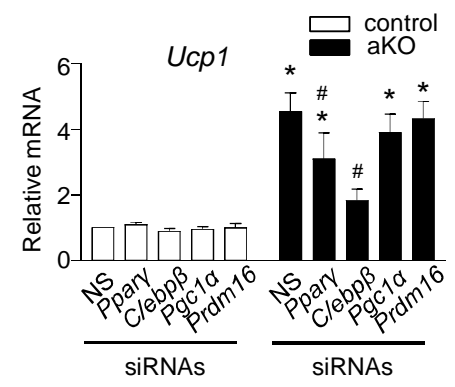

$\mathrm{H}$

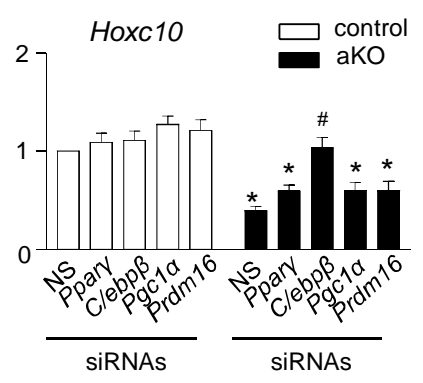

F

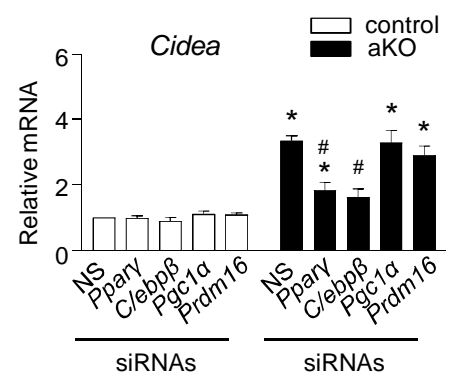

I

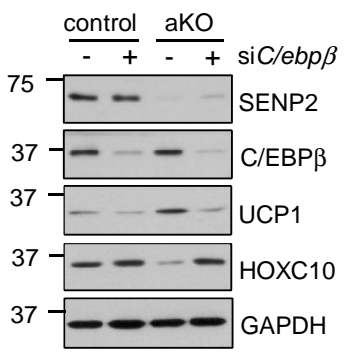




\section{Figure 6}

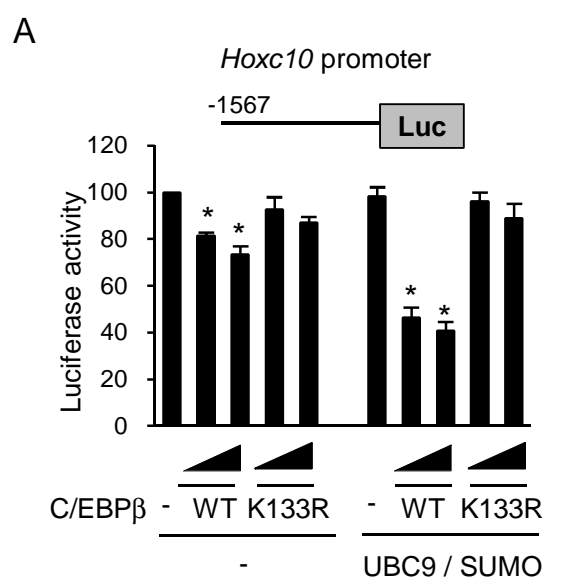

B

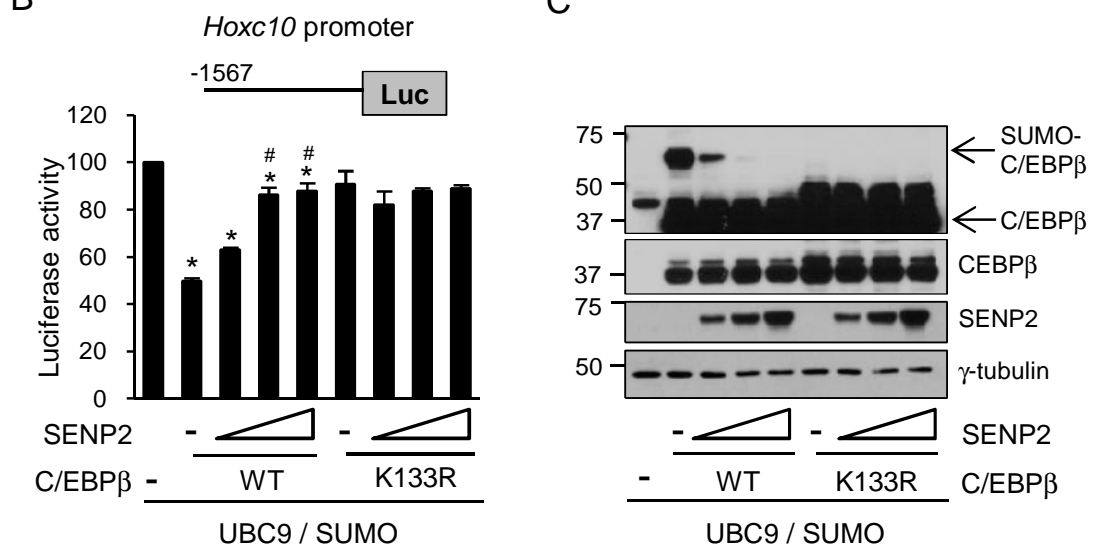

C

D

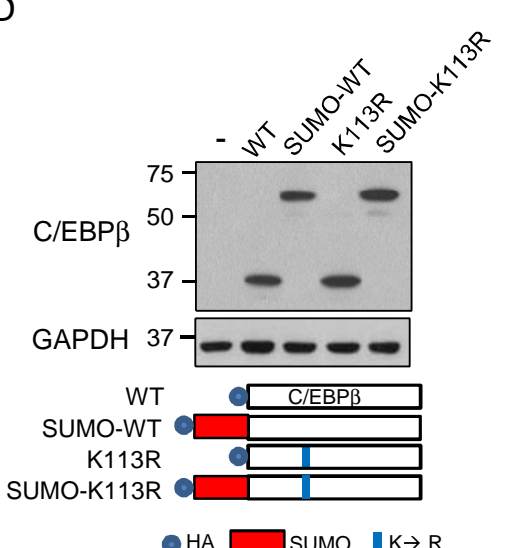

G
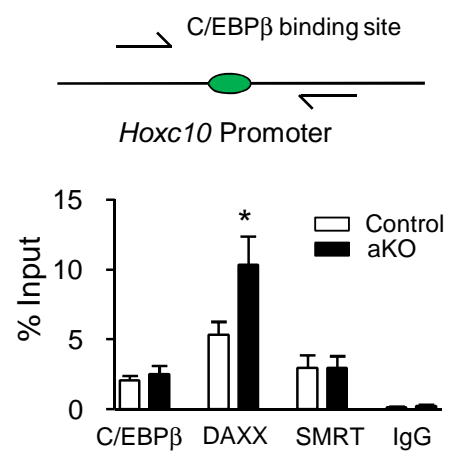

E

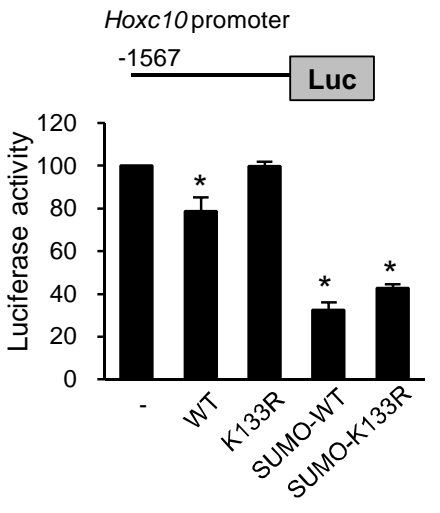

F Hoxc10 promoter

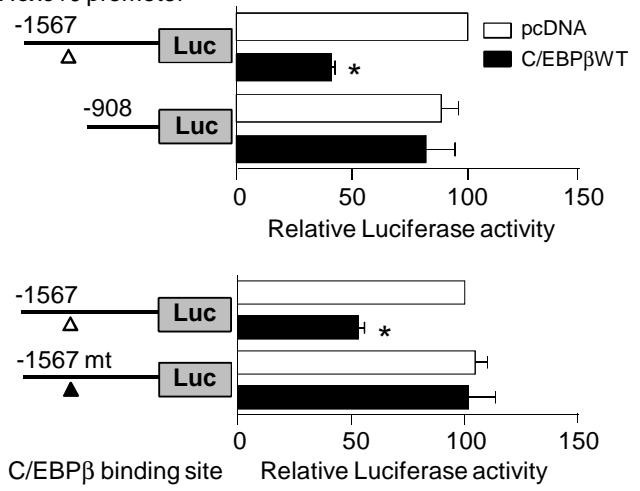

$\Delta \mathrm{C} / \mathrm{EBP} \beta$ binding site Relative Luciferase activity

$\mathrm{H}$

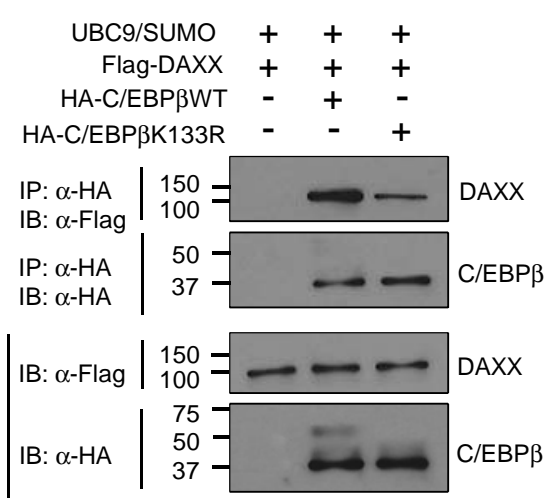

Control

S, SENP2 C/EBP $\longrightarrow$ Hoxc10 $\infty 000000$ SENP2-aKO

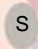
DAXX 7 , Hoxetg 00000000 


\section{Figure 7}

A

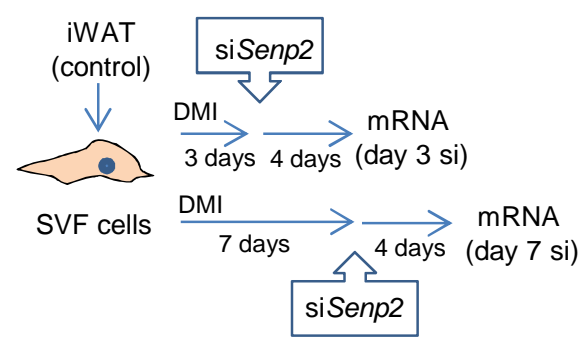

D

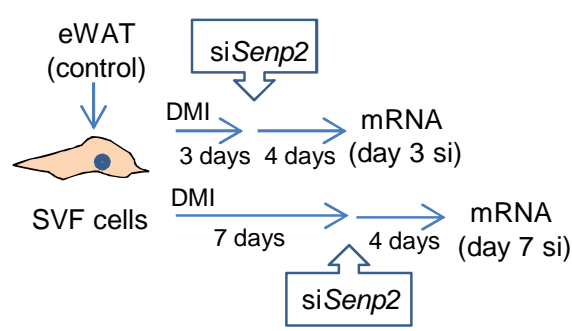

F

eWAT

(control, aKO)

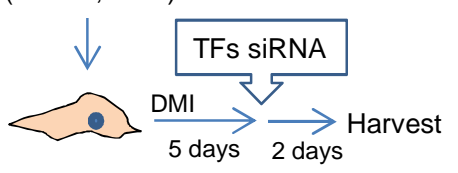

SVF cells

G
B

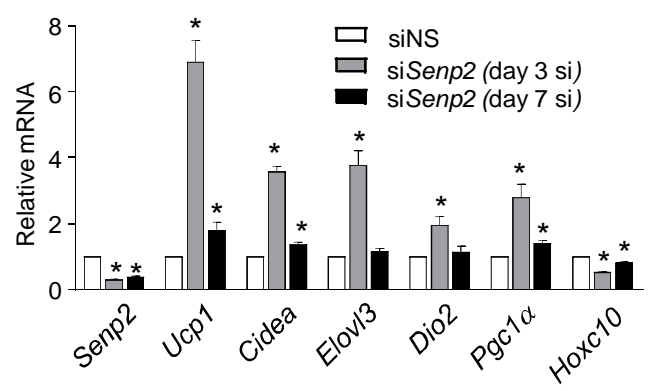

E
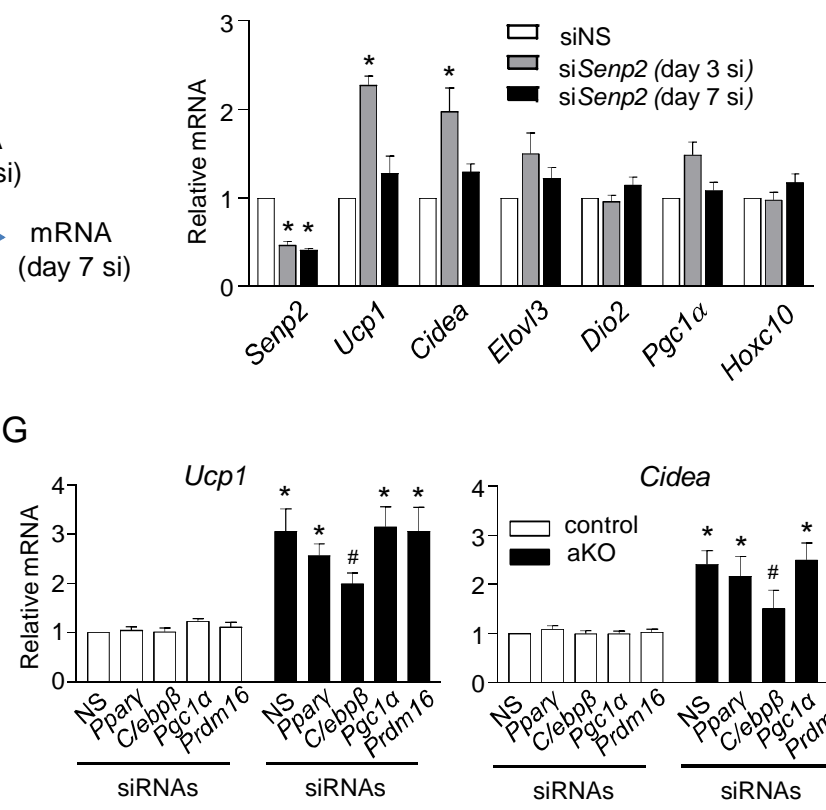

C

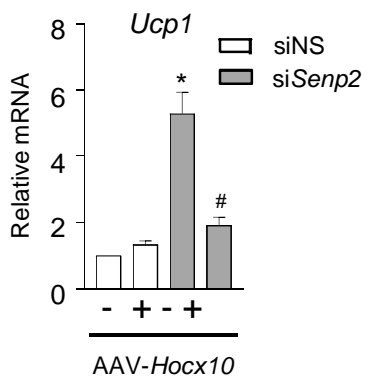

$\mathrm{H}$

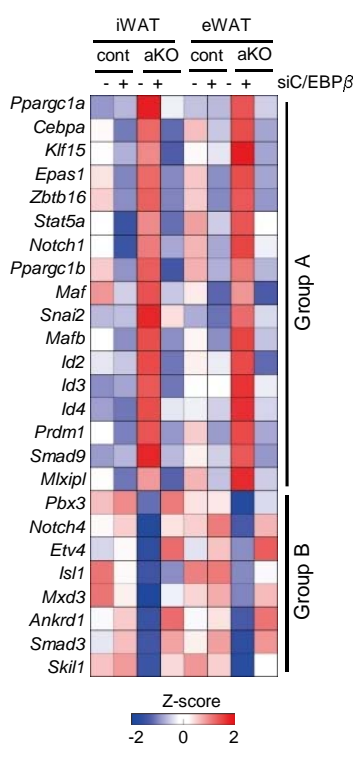

I
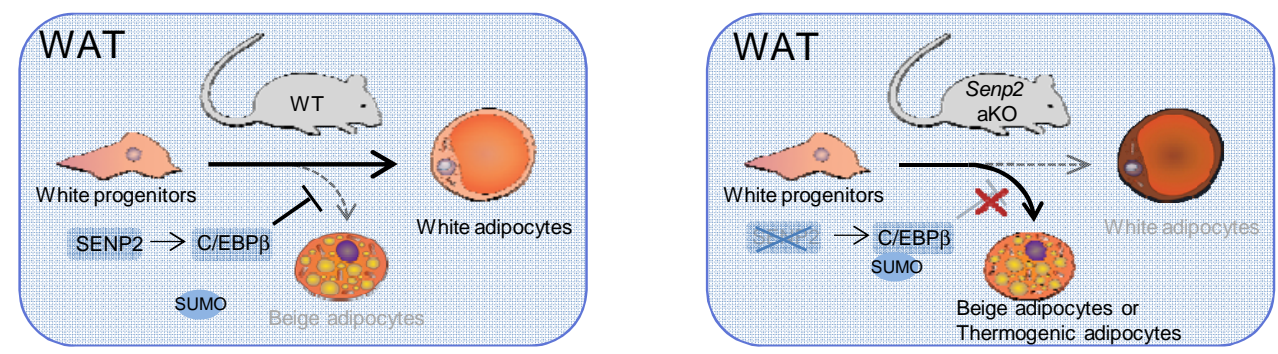